\title{
Control of rodent and human spatial navigation by room and apparatus cues
}

\author{
Derek A. Hamilton ${ }^{\mathrm{a}}$, Travis E. Johnson ${ }^{\mathrm{a}}$, Edward S. Redhead ${ }^{\mathrm{b}}$, Steven P. Verney ${ }^{\mathrm{a}}$ \\ ${ }^{a}$ Department of Psychology, University of New Mexico, Albuquerque, NM, USA 87131 \\ ${ }^{b}$ School of Psychology, University of Southampton, Southampton, UK SO17 1BJ
}

\begin{abstract}
A growing body of literature indicates that rats prefer to navigate in the direction of a goal in the environment (directional responding) rather than to the precise location of the goal (place navigation). This paper provides a brief review of this literature with an emphasis on recent findings in the Morris water task. Four experiments designed to extend this work to humans in a computerized, virtual Morris water task are also described. Special emphasis is devoted how directional responding and place navigation are influenced by room and apparatus cues, and how these cues control distinct components of navigation to a goal. Experiments 1-2 demonstrate that humans, like rats, perform directional responses when cues from the apparatus are present, while Experiment 3 demonstrates that place navigation predominates when apparatus cues are eliminated. In Experiment 4, an eyetracking system measured gaze location in the virtual environment dynamically as participants navigated from a start point to the goal. Participants primarily looked at room cues during the early segment of each trial, but primarily focused on the apparatus as the trial progressed, suggesting distinct, sequential stimulus functions. Implications for computational modeling of navigation in the Morris water task and related tasks are discussed.

Key words: place navigation, Directional responding, cognitive mapping, eyetracking, hippocampus
\end{abstract}

\section{Background and Introduction}

The experimental analysis of basic behavioral and learning processes involved in maze learning and spatial navigation has held a special place throughout the history of experimental psychology. Perhaps no single issue within the spatial navigation literature has been more intensely studied and debated than the question of what is learned when navigation to a 
particular place is reinforced. Tolman et al. (1946) contrasted place and response learning in the simple T-maze by training animals to either perform a particular response (e.g., always enter the arm to the right) or to navigate to a specific spatial location in the room regardless of the particular arm where reinforcement occurred. The ability of rats to learn where to navigate independently of specific motor responses represents what Tolman et al. (1946) termed a place disposition, a concept which became a central feature of Tolman's cognitive mapping theory (Tolman, 1948) and subsequently the influential mapping theory of O'Keefe and Nadel (1978). During the early years of this debate place learning and the place disposition were contrasted not only with the response disposition, but several alternative behavioral processes including approach/avoidance tendencies (Hull, 1943, 1934a,b), simple and complex guidance (Deutsch, 1960), and directional responding (Blodgett et al., 1949).

A growing body of recent literature has emphasized the relative contributions of place navigation and directional responding in rodent navigation tasks. The present paper has three aims: 1) To provide a brief review of the literature on directional responding and place navigation in the rat, 2) to present new empirical data on directional responding and place navigation in human participants, and 3) to discuss implications of the methodological approaches described here and the resulting data for computational modeling of navigation.

\subsection{Directional and Place Navigation in Dry Mazes}

Blodgett et al. (1949) noted that the apparent place disposition demonstrated by Tolman et al. (1946) might reflect learning to move in the direction of reinforcement within the room and maze rather than true place navigation. To contrast the relative influence of response, place, and directional strategies, Blodgett et al. rotated and/or repositioned a T-maze from trial to trial and systematically manipulated the reinforced arm such that only a single form of responding would reliably result in reinforcement. For the response only groups (groups III, VIII, and IX), reinforcement was always located in one arm of the maze (left or right), and because the maze was repositioned and rotated for each trial, the place and direction of reinforcement varied such that the particular turning response was the only response that reliably resulted in reinforcement. For the direction only groups (groups II and XI), reinforcement was always located in the same direction in the room and maze (e.g., to the east). The reinforced arm (left or right) and the precise location of reinforcement in the room varied from trial to trial, making the directional response the only response that always resulted in reinforcement. 
Finally, for the place only group (group I), reinforcement was always located the same place in the room regardless of the apparatus position. The reinforced arm (left or right) and the direction of reinforcement in the room and maze varied from trial to trial, thus, navigating to the same spatial location was the only response that always resulted in reinforcement. The groups for which a single directional or turning response reliably resulted in reinforcement made the fewest errors, whereas the place only group committed the most errors. Based on this outcome Blodgett et al. concluded that the relative contribution of place information to performance in the T-maze is negligible in comparison to response and directional information, thus, the apparent place disposition reported by Tolman et al. (1946) when the maze remained stationary can reasonably be explained in terms of directional responding rather than true place navigation. D. M. Skinner and colleagues (Skinner et al., 2003; Stringer et al., 2005; Horne et al., 2007) have recently replicated the findings of Blodgett et al. in the T-maze and reported similar observations in open field environments.

Olton et al. (1979) contrasted place navigation and orientation by pointing out that a place would be analogous to a single point (e.g., in a Cartesian coordinate system), whereas orientation involves a vector (a line and direction). Thus, navigating by moving in a particular direction within the apparatus and room can be thought of as occurring along an axis, or line, whereas place navigation involves movement to a particular point. The present evidence for directional responding is clearly consistent with Olton's characterization of orientation and inconsistent with the idea that rats preferentially learn to navigate to a single point.

\subsection{Directional Responding and Place Navigation in the Morris water task}

Over the past three decades the Morris water task (Morris, 1981, 1984; Sutherland and Dyck, 1984) has become a model behavioral task for the measurement of place navigation and learning. In this task, rats are trained to navigate to a hidden escape platform in a circular pool of cool, opaque water. The platform remains in the same spatial location and multiple release locations are used, thus, simple motor responses are ineffective and it is generally agreed that animals learn to navigate to the platform based upon its fixed spatial relationship to the available distal visual cues. The fact that the platform is in a fixed spatial relationship to distal visual stimuli, however, is neither sufficient to conclude that animals learn to navigate to a precise location in the water task, nor does it rule out the possibility that animals learn to move in the direction of the platform within the room and pool. 
To contrast directional responding and place navigation in the Morris water task, Hamilton et al. (2007) trained rats to swim to a hidden escape platform and then administered a single no-platform probe trial with the pool repositioned in the room such that the absolute spatial location of the platform in the room was centered in the opposite quadrant of the pool. For example, if an animal was trained with the pool in position 1 (see Fig. 1) and the platform in location B, moving the pool to position 2 for the no-platform probe trial could result in navigation to the absolute location of the platform (location B) or navigation in the direction of the platform in the room and apparatus (to the east), which would result in navigation to the relative location of the platform in the pool (location $\mathrm{C}$ ). The results clearly showed that rats swam in the direction of the platform and persisted in searching at the relative location rather than the absolute place where the platform was located. Further, animals treated the relative location as if it were the absolute (trained) location, while treating the absolute location as if it were an arbitrarily selected, untrained location. The studies by Hamilton et al. (2007; 2008b) have shown that directional responding predominates place navigation in wide range of procedural variants of the water task. The only condition in which a preference, albeit weak and transient, for place navigation over directional responding was observed involved filling the pool nearly to the top such that the pool wall was not a prominent feature of the environment (Hamilton et al., 2008a,b). Based on these findings, Hamilton et al. suggested that directional navigation in the standard water task involves distinct processes controlled by the distal room cues and apparatus cues (from the pool) or an interaction between the two sources of control. Specifically, they proposed that navigation to the relative location in the pool when the pool is repositioned involves a movement vector, in which the directionality of the trajectory is controlled by the distal room cues and subsequent search for the platform is controlled based on distance from the pool. An alternative explanation offered by Hamilton et al. (2008b) is that the distal cues disambiguate locations within the pool reference frame, thus, navigation to the relative location when the platform is repositioned occurs because subjects learn to navigate to a particular region of the pool based on the available room cues, rather than learning to navigate to a particular location within the room. The latter explanation is consistent with physiology data showing that hippocampal place cells tend to stay bound to the local, otherwise ambiguous, apparatus cues when the apparatus is translated in the room (Knierim and Rao, 2003; Yoganarasimha and Knierim, 2005). Nonetheless, these 
explanations and their related processes are not necessarily mutually exclusive and it may prove difficult to distinguish them experimentally. In either case, these explanations and the data that prompted them represent a departure from the generally accepted view that rats learn to navigate to a hidden escape platform in the Morris water task based on its fixed spatial relationship to the available distal room cues.

\subsection{Human navigation in a virtual Morris water task}

Over the last decade a number of laboratories have begun to measure spatial learning and navigation in humans through the use of virtual, computerized environments (Astur et al., 1998; Chamizo et al., 2003; Hamilton and Sutherland, 1999; Jacobs et al., 1998; Moffat et al., 1998; Redhead and Hamilton, 2007; Sandstrom et al., 1998). The precise control over the environment and behavior of participants and the ease with which such tests can be conducted has made the use of virtual environments increasingly popular. This methodology has proven useful for a variety of research problems including basic hypothesis testing with respect to behavioral and psychological processes and evaluation of spatial learning and navigation in patient and other special populations. Many of the specific protocols described above for rats can readily be adopted for human research. Here, we focus on a computerized (virtual) Morris water task (VMWT) developed in our laboratory (Brandt et al., 2005; Driscoll et al., 2003, 2005; Hamilton et al., 2002, 2003; Hamilton and Sutherland, 1999; Hufner et al., 2007; Schautzer et al., 2003) (See also Astur et al. (1998)). In this task, participants view the virtual environment from a first-person perspective and move through the environment using keyboard keys or a joystick. A representative view from within the virtual pool and a layout of a virtual environment used in the VMWT is shown in Figure 2. The virtual room consists of a circular pool and a collection of distal walls and cues. The platform is placed within the circular pool and can be either hidden or visible and in a fixed location or moving. Because the environment is completely controlled via a computer program it is possible to manipulate the environment in ways that are difficult or impossible to do with real environments. Further, because participants do not move physically change location as they do in real navigation the major source(s) of stimulus control are limited to exteroceptive visual stimuli. Like rats, humans that have mastered the task take direct paths to the hidden platform from several release points around the perimeter of the pool and will persist in searching at the platform location when the platform is removed for a probe trial. In addition to these similarities, several reports 
have demonstrated that humans respond to manipulations involving apparatus and/or room cues in much the same way as rats (Hamilton et al., 2002; Hamilton and Sutherland, 1999; Redhead and Hamilton, 2007), suggesting basic similarities in how room and apparatus cues control human and rodent navigation in their respective tasks. An evaluation of the relative contributions of place navigation and directional responding in the virtual Morris water task has not been conducted.

The experiments reported here utilized the VMWT to investigate the control of human navigation by room and apparatus cues. Experiment 1 was undertaken to determine if humans display a preference for directional responding over place navigation in the VMWT. Experiments 2 and 3 evaluated the effects of reducing or eliminating the pool as a source of control. In Experiment 4, moment to moment eye location on the display was measured and co-registered with moment to moment navigation data, thus providing a dynamic record of where participants looked in the virtual environment as they navigated from the release point to the platform location. The resulting data were used to inform whether control by room and pool cues can be distinguished, particularly with respect to their relative contributions to control of navigation at various stages of an individual trial as suggested by Hamilton and colleagues (Hamilton et al., 2007, 2004).

[Figure 1 about here.]

[Figure 2 about here.]

\section{Experiment 1}

The goal of Experiment 1 was to evaluate whether the basic preference for directional responding over place navigation observed in rats in the Morris water task is also observed in humans in a virtual Morris water task. Participants were trained to navigate to a hidden escape platform and given a single no-platform probe trial with the pool repositioned in the virtual room.

\subsection{Method}

\subsubsection{Participants}

Participants were undergraduate students enrolled in psychology courses at the University of New Mexico who received course credit for participating. There were 12 female and 12 male 
participants who were 18-37 years of age and had not previously participated in virtual navigation experiments conducted in our laboratory. All participants provided informed consent prior to participating. None of the participants reported a history of neurological disorders and all participants had normal or corrected vision. All procedures were approved by the Institutional Review Board (IRB) at the University of New Mexico.

\subsubsection{Apparatus}

The virtual environment consisted of a circular pool located in a room with a square floorplan. Fig. 2 shows a scale layout of the environment and a representative first-person view of the environment from a participant's perspective. We adopt the convention of describing the relative sizes of the components of the environment and distances in virtual space in arbitrary units. The virtual room was 16 units in width and length and 3 units in height. The distal room walls were visually identical with the exception of distinct, abstract visual cues located on each of the four distal walls. The cues were 3 units X 3 units and provided the only features of the environment that could disambiguate locations. The circular pool was 3.2 units in diameter with a perimeter wall that extended approximately .66 units above the surface of the pool. The position of the pool within the room varied and is specified for each experiment. The square platform was approximately .66 units in width and length and extended approximately .33 units above the surface of the pool. Auditory feedback consisted of a bell which sounded when the platform was located, an aversive, discordant tone which sounded if the trial duration exceeded $60 \mathrm{sec}$, and the sound of moving water which accompanied forward movement through the pool. An IBM-compatible computer controlled the presentation of the environment, auditory feedback, and data collection. Visual aspects of the experiment were displayed on a 17 in. color monitor. Navigation was controlled using the keyboard arrow keys. The UP arrow key was used to control forward movement while the LEFT and RIGHT arrow keys controlled rotation. Backward navigation was not possible. Direct traversal of a distance equal to the diameter of the pool took approximately 4 sec to complete and a full rotation in the absence of forward movement took approximately 2.5 sec.

\subsubsection{Design and Procedure}

After providing informed consent participants were given instructions by the experimenter. The instructions included a description of the task and how to navigate using the keyboard 
keys, however, no information regarding strategies or experimental manipulations was provided. Experiment 1 was conducted in two phases. During phase 1 (training), all participants were given 5 blocks of 4 hidden platform trials. For 12 participants (6 male and 6 female) the pool was in position 1 and for the remaining 12 participants the pool was in position 2 (see Fig. 2). The escape platform was at location B for both pool positions. Escape latency and total path length were measured for each training trial. Previous research from our laboratory has shown that $20 \%-35 \%$ of subjects fail to consistently take direct trajectories to the platform from the various release points. Each subject's "swim" paths during the final training trial block were inspected by one observer and classified as direct if the subject took direct trajectories during each of the 4 trials or non-direct otherwise.

Phase 2 consisted of a single 60 sec probe trial with the platform removed from the pool. An equal number of participants for each pool position used during training were randomly assigned to No Shift and Shift groups, however, only data from subjects classified as taking direct trajectories during training were included in the probe analyses. For participants in the No Shift group the pool remained in the same position used during training. For participants in the Shift group the pool was relocated to the position that was not used during training. Four dependent measures were taken for each of two critical locations that were the same size as the platform surface. One critical location was the absolute location of the platform in the room and the other was an equal distance from the pool wall in the diametrically opposite quadrant. When the pool is repositioned, the opposite location corresponds to the same relative location of the platform in the pool during training, and is the location to which a directional response would be expected. If the pool is not repositioned, the opposite location serves as a comparison location which has the same spatial relationship to the absolute location as does the relative location for conditions in which the pool is repositioned. For example, if the pool was in position one and the platform was in location B (see Fig. 2) during training, and the pool remained in position one for the probe trial, the opposite location would correspond to location A. If the pool was repositioned (to position 2) for the probe then location B corresponds to the absolute location and location $\mathrm{C}$ corresponds to the relative/opposite location. The number of times each critical location was crossed and the average distance from each location during the probe trial were measured. The latter measure was adapted from the goal proximity measure described by Gallagher et al.. The latency to enter and the amount of time spent in a circular 
region (1 unit in diameter) centered around each of the critical locations were also measured Akers et al. (2007); Hamilton et al. (2007, 2008b,a). Release points for the probe trial were selected pseudorandomly from two locations (NW, SE) that were equidistant from the two critical comparison regions.

\subsection{Results and Discussion}

All effects were significant at $p<.05$ unless otherwise noted.

\subsubsection{Hidden platform training}

A total of 18 out of the 24 participants learned to take direct trajectories to the platform from each of the four release points by the final training block. The remaining 6 participants took circuitous or otherwise non-direct trajectories throughout the training trials. The proportion of participants who failed to take direct trajectories to the platform was equally distributed among the to be probe conditions (3 Shift and 3 No Shift) and sex (3 male and 3 female), and is consistent with previous observations using this task (e.g., Hamilton and Sutherland (1999); Hamilton et al. (2002)). Subsequent training and probe analyses are limited to the 18 participants who learned to take direct trajectories during training.

Mean latencies and path lengths for each of the five training trial blocks were calculated and analyzed in separate repeated measure analyses of variance (ANOVAs) with Trial Block (repeated with 5 levels), sex, and the to-be probe condition (Shift or No Shift) as factors. There were significant main effects of Trial Block for latency, $F(4,56)=16.67$, and path length, $F(4,56)=13.87$ (data not shown), both of which resulted from significant linear decreases across training trial blocks, both $p \mathrm{~s}<.001$. Higher-order Trial Block effects failed to reach significance. There were no significant differences in performance related to sex or to be probe condition, all $p \mathrm{~s}>.17$, and there were no significant interactions, all $p \mathrm{~s}>40$.

\section{[Figure 3 about here.]}

[Figure 4 about here.]

\subsubsection{Shift vs. No-Shift Probe Trial}

Analyses of the no-platform probe trial data were limited to the 18 participants who were classified as taking direct trajectories to the platform during training. Representative

probe trial "swim" paths for participants from each group are shown in Fig. 3. All 9 of the 
participants in the No Shift group navigated directly to the absolute region during the probe trial. In contrast, 7 of the 9 participants in the Shift group navigated directly to the relative location (1 navigated directly to the absolute location, and the other did not show a clear preference for either location). This pattern is consistent with the rodent data described above and suggests that directional responding predominates navigation to the absolute location of the platform within the room reference frame in human virtual navigation.

Separate analyses of variance (ANOVA) for each probe trial dependent measure were conducted with group (Shift vs. No Shift) and sex as between-subjects factors and location (Absolute vs. Relative/Opposite) as a within-subjects factor. Group means for each location and dependent measure are shown in Fig. 4. None of the interactions involving sex or the sex main effect were significant, all $p \mathrm{~s}>.12$. For time in region there were main effects of location (Absolute $>$ Relative/Opposite), $F(1,14)=10.22$, and group (No Shift $>$ Shift), $F(1$, $14)=7.18$, and there was also a significant main effect of location for the proximity measure (Absolute $<$ Relative/Opposite), $F(1,14)=13.56$. The primary tests of interest are the Location X Group interactions, which were significant for latency to enter to the critical regions, $F(1,14)=45.35$, time spent in the critical regions, $F(1,14)=38.12$, number of times each critical location was crossed, $F(1,14)=11.62$, and proximity to the critical locations, $F(1,14)=70.77$. Analyses of simple main effects revealed that participants in the No Shift group entered the absolute region faster than the opposite location, $F(1,8)=70.38$, spent more time in the absolute region, $F(1,8)=44.79$, crossed the absolute location more frequently, $F(1,8)=17.06$, and, on average, navigated closer to the absolute location, $F(1$, $8)=182.04$. Subjects in the Shift group entered the relative location faster than the absolute location, $F(1,8)=8.92$, and navigated closer to the relative location, $F(1,8)=5.85$. Although subjects in the Shift group crossed the relative location more than the absolute location and spent more time in the relative region compared to the absolute region, neither of these effects reached significance, both $p \mathrm{~s}>.07$.

\subsubsection{Summary}

The results of Experiment 1 demonstrate that humans in a virtual Morris water task, like rats in the Morris water task, navigate in the direction of the platform within the room and apparatus rather than navigating to the precise location of the platform in the room. Thus, the results of Experiment 1 expand the generality of the basic preference for directional 
responding reported in the Morris water task to include humans.

\section{Experiment 2}

Recently, Hamilton and colleagues Hamilton et al. (2008b,a) demonstrated that rats display a preference for place navigation over directional responding when the size of the pool wall was reduced. This was achieved by filling the pool nearly to the top; The water surface was a few centimeters below the top of the pool in order to prevent escape, thus, only a small amount of the pool wall remained visible. Experiment 2 was designed to determine whether a similar preference for place navigation is observed in humans trained and tested under comparable conditions in a virtual Morris water task.

\subsection{Method}

\subsubsection{Participants}

Participants were 12 males and 12 females from the same population as described in Experiment 1.

\subsubsection{Apparatus}

Two environments were used in Experiment 2. One environment was used only in a pretraining phase. The pretraining environment was similar to the environment used for subsequent training and testing phases, but contained and visible pool wall (.66 units in height) and 4 novel distal cues that were only present during the pretraining phase. The second environment which used for the primary training and testing phases was the same as that used in Experiment 1 with the exception that the perimeter pool wall was only .01 units in height rather than .66 units as in Experiment 1 and pretraining.

\subsubsection{Design and Procedure}

A pretraining phase using a novel environment was included in Experiment 2 for two reasons. First, Hamilton et al. (2008a) demonstrated that a preference for place navigation over directional responding is observed only early in training when the pool wall is reduced as a source of control, thus, it was important to only give participants minimal training with a small pool prior to testing. Second, considerable previous work from our laboratory (see e.g., Driscoll et al. (2005); Hamilton and Sutherland (1999); Hanlon et al. (2006)) indicates that participants generally need 8 - 16 trials in order to learn all the basic features of the 
task in addition to learning to take direct trajectories to the platform. To address both issues, participants were first given 4 blocks of 4 hidden platform trials in the pretraining environment, in which the pool wall was the same height as that used in Experiment 1. Subsequent training in the second environment, which contained a pool wall only .01 units in height, consisted of a single block of 4 trials. A single no-platform probe trial with the pool repositioned (Shift) or in the same position as used in training (No Shift) was conducted.

\subsection{Results and Discussion}

\subsubsection{Hidden platform training}

A total of 13 out of the 24 participants learned to take direct trajectories to the platform by the third and fourth trials of the final training block. The remaining 11 participants took circuitous or otherwise non-direct trajectories throughout the final trial block. The proportion of participants who failed to take direct trajectories to the platform was roughly equally distributed among the to be probe conditions (5 Shift and 6 No shift), however, a greater number of females failed to meet criteria (4 male and 7 female). Subsequent training and probe analyses are limited to the 13 participants who learned to take direct trajectories during training.

Mean latencies and path lengths for each training trial during the blocks were calculated and analyzed in separate repeated measure analyses of variance (ANOVAs) with Trial (repeated with 4 levels), sex, and the to-be probe condition (Shift or No Shift). There were significant main effects of Trial for latency, $F(3,27)=3.20$, and path length, $F(3,27)=$ 5.93 (data not shown), both of which resulted from significant linear decreases across training trial blocks, both $p$ s <.03. Higher-order Trial effects failed to reach significance. Importantly, subjects who learned to take direct trajectories to the platform during the final training phase reached comparable asymptotic levels of performance to that observed in Experiment 1. There were no significant differences in performance related to sex or to be probe condition, all $p \mathrm{~s}$ $>.32$, and there were no significant interactions, all $p \mathrm{~s}>079$.

[Figure 5 about here.]

\subsubsection{Shift vs. No Shift probe trial}

Analyses of the no-platform probe trial data were limited to the 13 participants who were classified as taking direct trajectories to the platform during training. Representative 
probe trial "swim" paths for participants from each group are shown in Fig. 5. All 6 of the participants in the No Shift group navigated directly to the absolute region during the probe trial. In contrast, all 7 of the participants in the Shift group navigated directly to relative location.

[Figure 6 about here.]

Separate analyses of variance (ANOVA) for each probe trial dependent measure were conducted with group (Shift vs. No Shift) and sex as between-subjects factors and location (Absolute vs. Relative/Opposite) as a within-subjects factor. Group means for each location and dependent measure are shown in Fig. 6. Males crossed the critical locations significantly more often than females, $F(1,9)=10.24$, and there was also a Location X Sex interaction for the number of times each location was crossed, $F(1,9)=5.40$. Although there were effects involving the sex factor, we note that the basic pattern of results was the same for males and females. Therefore, we do not pursue further analyses of the sex effects. None of the other main effects or interactions involving sex were significant. There were significant main effects of group for latency to enter critical regions (No Shift $<$ Shift), $F(1,9)=17.29$, and proximity to the critical locations (No Shift $<$ Shift), $F(1,9)=6.09$. None of the other main effects for group were significant, all $p \mathrm{~s}>.06$. As with the analyses for Experiment 1, the primary tests of interest are the Location X Group interactions, which were significant for latency to enter to the critical regions, $F(1,9)=105.47$, time spent in the critical regions, $F(1,9)=53.77$, number of times each critical location was crossed, $F(1,9)=14.20$, and proximity to the critical locations, $F(1,9)=67.78$. Analyses of simple main effects revealed that participants in the No Shift group entered the absolute region faster than the opposite location, $F(1,5)=88.03$, spent more time in the absolute region, $F(1,5)=25.66$, crossed the absolute location more frequently, $F(1,5)=7.66$, and, on average, navigated closer to the absolute location, $F(1,5)=32.84$. Participants in the Shift group entered the relative location faster than the absolute location, $F(1,6)=30.35$, spent more time in the relative region compared to the absolute region, $F(1,6)=27.30$, and navigated closer to the relative location than the absolute location, $F(1,6)=19.71$. Participants in the Shift group crossed the relative location more than the absolute location this effect was not significant. 


\subsubsection{Summary}

The results of Experiment 2 suggest that decreasing the height of the pool wall does not lead to a preference for place navigation over directional responding in human virtual navigation. Based on the available data from the Morris water task, we expected that reducing the height of the pool wall would result in a preference for place navigation over directional responding. Together with the results of Experiment 1, the findings from Experiment 2 suggest that directional responding predominates place navigation in human virtual navigation independently of the prominence of apparatus cues. While the basic pattern of data was the same for Experiments 1 and 2, the reduction in the pool wall did reduce the number of participants who learned to take direct trajectories during training. With a prominent pool wall in Experiment 1, about $75 \%$ of participants learned to take direct trajectories, whereas only $54 \%$ took direct trajectories in Experiment 2. Participants in Experiment 2 only received 4 trials in the training environment compared to 20 trials for the participants in Experiment 1, however, pilot data for participants who were given 20 training trials with the reduced pool wall revealed a similar proportion of participants who took direct trajectories (50\%). This further indicates that the pool wall is important determinant of behavior, even though it does not disambiguate spatial locations. This observation is somewhat curious, in that it might be expected that reducing the pool wall would increase visibility of the distal room cues and, therefore, improve performance. We note that this observation is consistent with findings in the rat Hamilton et al. (2008b,a) in which rats learn to navigate with a reduced pool wall at a slower rate compared to rats trained with a prominent pool wall.

\section{Experiment 3}

Experiment 3 was designed to evaluate whether a preference for place navigation is observed when the pool wall is completely eliminated as a source of control. Complete elimination of the pool is not possible in real world environments, thus, this experiment took advantage of the capabilities of virtual navigation procedures to address a question that cannot be unambiguously addressed in real world navigation experiments. The critical training and testing procedures were the same as those used in Experiment 1 with the exception that the wall of the virtual pool was not visible. 


\subsection{Method}

4.1.1. Participants

Participants were 12 females and 12 males from the same population as described in Experiment 1.

\subsubsection{Apparatus}

Two environments were used in Experiment 3. One environment was used only for pretraining and was the same as the pretraining environment used in Experiment 2. The second virtual environment which was used for the primary training and testing phases was the same as that used in Experiment 1 with the exception that the perimeter wall of the pool was not visible.

\subsubsection{Design and Procedure}

Instructions were the same as those used in the previous experiments with the exception that participants were informed that the pool wall would be present but not visible during some parts of the experiment. This modification was done to reduce confusion regarding why forward movement might stop in the absence of any visible obstacles. Pilot work revealed that participants have considerable difficulty learning to navigate within a pool that is not visible, spending much of their time colliding with the invisible pool wall. A set of two pretraining trials blocks with a visible pool wall were given in order to provide participants with training in the basic features of the task prior to training with the invisible pool wall. Pretraining was conducted in an environment that was not used in subsequent training and the pool was located in the center of the virtual room. After pretraining, participants were given hidden platform training trials and a no-platform probe trial following the same procedures used in Experiment 1 with the exception that the perimeter pool wall was not visible. Participants were given 5 blocks of 4 hidden platform trials with the pool either in location A or B (see Fig. 2). A single no-platform probe trial was conducted with pool repositioned (Shift) or in the same position used during training (No Shift).

\subsection{Results and Discussion}

\subsubsection{Hidden platform training}

A total of 8 out of the 24 participants learned to take direct trajectories to the platform by the final training block. The remaining 16 participants took circuitous or otherwise non-direct 
trajectories throughout the final trial block. The proportion of participants who failed to take direct trajectories to the platform was equally distributed among the to be probe conditions ( 8 shift and 8 no-shift) and sex (8 male and 8 female). Subsequent training and probe analyses are limited to the 8 participants who learned to take direct trajectories during training.

Mean latencies and path lengths for each training trial during the final trial block were calculated and analyzed in separate repeated measure analyses of variance (ANOVAs) with Trial (repeated with 4 levels) and the to-be probe condition (Shift or No Shift). Because there were only two males and females in each of the probe conditions sex was not included as a factor in the analyses. There were significant main effects of trial block for latency, $F(4$, $24)=5.70$, and path length, $F(4,24)=5.32$ (data not shown), both of which resulted from significant linear decreases across training trial blocks, both $p \mathrm{~s}<.02$. Higher-order Trial effects failed to reach significance. There were no significant main effects of probe condition or Trial Block X Probe Condition interactions.

[Figure 7 about here.]

\subsubsection{Shift vs. No Shift probe trial}

Analyses of the no-platform probe trial data were limited to the 8 participants who were classified as taking direct trajectories to the platform during training. Representative probe trial "swim" paths for participants from each group are shown in Fig. 7. All 4 participants in the No Shift and Shift groups navigated directly to the absolute region during the probe trial. This pattern is consistent with rodent data in which a preference for place navigation over directional responding is observed when the pool is reduced in size, and is in contrast with the results of Experiments 1 and 2 reported here which found that directional responding predominates place navigation regardless of the pool's prominence in the environment.

[Figure 8 about here.]

Separate analyses of variance (ANOVA) for each probe trial dependent measure were conducted with group (Shift vs. No Shift) as a between-subjects factor and location (Absolute vs. Relative/Opposite) as a within-subjects factor. Group means for each location and dependent measure are shown in Fig. 8. The were significant location effects for latency (Absolute < Relative/Opposite), $F(1,6)=22.90$, time in region (Absolute $>$ Relative/Opposite), $F(1$, 
$6)=13.72$, location crosses (Absolute $>$ Relative/Opposite), $F(1,6)=9.00$, and average proximity (Absolute $<$ Relative/Opposite), $F(1,6)=48.04$. None of the group main effects or interactions were significant, all $p \mathrm{~s}>.21$.

\subsubsection{Summary}

The results of Experiment 3 indicate that place navigation predominates directional responding in human virtual navigation when the pool wall is completely absent as a source of control. Further, only $33 \%$ of participants learned to take direct trajectories to the platform, even though the conspicuous distal environmental cues were just as visible as the cues from Experiment 2, and actually more visible than the cues in Experiment 1 which were partially obscured by the pool wall. This set of observations suggest the counter-intuitive conclusion that increasing the amount dependence of navigation on distal room cues has a detrimental effect on navigation. Taken with the results of Experiments 1 and 2, the results of Experiment 3 further indicate the importance of the pool wall as a source of control.

\section{Experiment 4}

Hamilton and colleagues have suggested the distal room cues and pool wall controlled two distinct, sequential components of rodent navigation in the Morris water task. The distal cues appear to control the initial trajectory in the direction of the platform Hamilton et al. (2007) (see also Hamilton et al. (2004)), while searching at the location of the platform was suggested to be controlled by the apparatus, with the distance to the apparatus wall being of particular importance. Given the similarity of the rat data reported by Hamilton et al. and the human data reported in Experiment 1, Experiment 4 was undertaken to evaluate whether the basic control provided by room and apparatus cues suggested by Hamilton et al. can be quantified in human participants. To do this, eye tracking equipment was used to estimate the location of eye fixations in the virtual environment dynamically as participants navigated to the escape platform.

\subsection{Method}

\subsubsection{Participants}

Participants were 13 male and 24 female volunteers from the same population as described in Experiment 1. 


\subsubsection{Apparatus}

The virtual environment was the same as the environment used in Experiment 1, with the exception that the pool was positioned in the center of the virtual room and the escape platform was located in the center of the NW quadrant of the pool. Measurement of gaze location on the computer screen were made using an EyeLink II head-mounted eyetracking system (SR Research Ltd., Osgoode, ON, Canada) The EyeLink II eyetracking system is a real-time corneal-reflection system with an average gaze position error of less than 0.5 degrees and high resolution of 0.025 degrees. One personal computer controlled the presentation of the virtual environment and a separate computer controlled acquisition and storage of eyetracking data.

\subsubsection{Design and Procedure}

Participants were seated in a dimly lit room in front of a $17 \mathrm{in.} \mathrm{computer} \mathrm{monitor} \mathrm{and}$ keyboard. The head-mounted eyetracking cameras and infrared lights were placed on the participant's head and adjusted to fit snugly but comfortably. The experimenter positioned and focused the cameras to achieve pupil area measurements (from the EyeLink system) of at least 75 and corneal reflection measurements of at least 95 for each eye as suggested by the manufacturer. The spatial location of the individual eye gaze locations on the computer display was calibrated using a standard $3 \mathrm{X} 3$ grid protocol which requires participants to fixate a small circle located at each of 9 positions distributed across the display.

The virtual navigation task consisted of a training phase ( 5 blocks of 4 hidden platform trials each) and a single no-platform probe trial. To correct for drift in the eyetracking locations across trials, prior to each trial participants were asked to fixate on a single small circle the center of the display. During training participants were released at each of 4 locations $(\mathrm{N}, \mathrm{S}, \mathrm{E}, \mathrm{W})$ in a pseudorandom sequence within each trial block. A single no-platform probe trial (60 sec) was conducted at the end of the training phase (note that the pool was not repositioned for the probe trial). The participant's position in $x, y$ coordinates and heading in the virtual environment were measured and recorded at $10 \mathrm{~Hz}$. The position of the participant's gaze on the display was recorded at $250 \mathrm{~Hz}$, and event markers (e.g., trial onset, finding the platform) generated by the program that controlled the virtual navigation protocol were inserted into the record of eye positions for subsequent co-registration of the virtual navigation and eyetracking data. 
Analysis of the eyetracking and virtual navigation data was conducted offline after the experimental session was completed. The left and right eye locations were averaged for each observation to produce a single eye location measurement. Eye locations that were outside the boundaries of the display were rare and were excluded. A separate program recreated the virtual environment and display using the moment-to-moment position and heading data. To determine the focus of the participant's gaze in 3-dimensional virtual space, the program utilized the transformation matrix used by the virtual navigation program to convert the 3-dimensional environment to a 2-dimensional matrix for display. The virtual environment was created by drawing a series of individual square segments to form walls, the pool surface, and cues. Because the conversion from 3-d to 2-d space is always the same for a particular combination of heading and position in virtual space, determining the 3-d position of the eye position in virtual space can be achieved by finding the particular segment of the environment that was drawn to the 2-d coordinates corresponding to the observed eye position.

For each trial, the program generated a "swim" path, an ethogram of eye locations, and a "dwell" plot. The swim paths were used to determine whether a participant learned to take direct trajectories to the platform. For analysis purposes, the environment was separated into 8 regions: 4 quadrants (NW,NE,SE,SW) for the distal room environment (i.e., beyond the pool wall) and within the pool. For each sample of each trial the region where the eye position was localized in the virtual environment was recorded and represented in an ethogram (see below). From these data, the overall proportion of time the participant's gaze was in each region for a given trial was also determined. Dwell plots were created by dividing the environment into discrete regions of equal size $(0.25 \mathrm{X} 0.25$ units $)$ and representing the total amount of time spent looking at each region over the entire trial (see probe trial analyses below).

\subsection{Results and Discussion}

[Figure 9 about here.]

\subsubsection{Hidden Platform Training}

Inspection of the swim paths generated for the training trials, 23 of the 37 subjects learned to take direct trajectories to the platform from all four release points by the final training block, while the remaining 14 participants took non-direct trajectories. The number of male and female participants in each category was roughly equivalent. 
Representative ethograms for participants classified as direct and non-direct for each of the final four training trials are shown in Fig. 9. Participants characterized as direct tended to look primarily at the distal room environment during the early portions of training trials and switched to primarily looking inside the pool, particularly at the region of the pool where the platform was located, during the later portions of the trial (the supplementary video shows the location of the eyes superimposed on the display for single trial during the final trial block by a direct participant). In contrast, participants classified as non-direct tended to look primarily in the pool throughout most of the trial, with no particular preference for any region of the pool. Although non-direct participants looked at the distal room early during the trials, they did so to a far lesser degree than direct participants. To evaluate these impressions, an analysis was conducted on the percentage of time spent looking at room and pool cues as a function of the portion of the trial (from release to finding the platform). Each trial was broken down into 20 segments based on the overall trial duration, which was variable based on how quickly the participant found the platform. The percentage of time spent looking at room cues or inside the pool were calculated individually for each segment. The means for each segment during the final block of trials was computed (see Fig. 10) and analyzed in a model ANOVA with proportion of the swim path (20 levels), region (Room vs. Pool) and classification (Direct vs. Non-direct) as factors. All interactions and main effects were significant, all $p \mathrm{~s}<.001$. Analyses conducted for each classification revealed that direct participants looked at the distal room cues significantly more than the pool over the first through fifth segments, but looked at the pool more than the distal room cues from seventh through final segment, all $p \mathrm{~s}<.001$. In contrast, non-direct participants looked at the distal room more than the pool during the first segment, but looked at the pool more than the room cues in every segment thereafter, all $p \mathrm{~s}<.001$. These observations indicate that participants who learned to take direct trajectories can be distinguished from those who do not on the basis of patterns of changes in the types of cues participants look at during navigation. More importantly, the shift from looking primarily at room cues to looking primarily at pool cues in direct participants is consistent with claims that distal room cues control the selection of a trajectory during the early stages of a trial in the Morris water task (Hamilton et al. (2004, 2007); Sutherland and Hamilton (2004)), whereas apparatus cues control subsequent aspects of navigation such as searching at the appropriate distance from the pool wall (Hamilton et al. 
$(2007,2008 b))$.

Inspection of the individual ethograms for direct participants also revealed considerable variability in the number of distal room regions that participants viewed across trials. Every direct participant viewed at least 1 and up to 3-4 distal room regions during the 4 trials of the final trial block. This is reflected in the direct participants' ethogram shown in Fig. 9. This is important in that it suggests that an accurate trajectory can be achieved with only minimal sampling of the distal room environment in a participant that has mastered the task.

[Figure 10 about here.]

\subsubsection{No-platform Probe Trial}

[Figure 11 about here.]

Representative ethograms and dwell plots for the probe trial are shown in Fig. 11. The percentage of time spent in each pool quadrant during the probe trial for direct and non-direct participants (see Fig. 12A) was analyzed with classification (Direct vs. Non-direct) and pool region (NW,NE,SE,SW) as factors. There was a significant interaction, $F(3,105)=22.74$, and a significant main effect of region, $F(3,105)=26.65$. Direct participants spent more time in the target quadrant $(\mathrm{NW})$ compared to the other quadrants, all $p \mathrm{~s}<.001$, whereas no significant quadrant differences were observed for non-direct participants, all $p \mathrm{~s}>.36$. Direct participants spent significantly more time in the target quadrant $(\mathrm{NW}+)$ than non-direct participants, $F(1,35)=35.99$. Inspection of the individual ethograms and dwell plots suggested that direct participants primarily looked at the region of the pool where the platform was located rather than looking primarily at the distal room cues. In contrast, non-direct participants looked primarily in the pool, however, the distribution of eye positions appeared to be more evenly distributed across the other, non-target regions of the pool. To evaluate these impressions, the percentage of time spent looking at each of the 4 quadrants (NW+,NE,SE,SW) of the pool and room (see Fig. 12B) were analyzed using a mixed model ANOVA with Region (Pool vs. Room), Quadrant (NW+,NE,SE,SW) and Classification (Direct vs. Non-direct) as factors. All of the main effects and interactions were significant, all $p \mathrm{~s}<.03$.

To better understand the pattern of eye location results, separate ANOVAs were conducted with Region and Quadrant as factors for direct and non-direct participants. Both direct and non-direct participants spent the clear majority of time looking within the pool, both $p$ s $<$ 
.001. There were significant location effects for direct and non-direct participants, both $p \mathrm{~s}<$ .001 , that were attributable to the fact that participants looked in the target quadrant more than the other quadrants. Inspection of the means for each combination of region and location suggests that participants discriminated among the quadrants of the pool, but not the distal room quadrants. To evaluate this impression for direct and non-direct participants a single target quadrant preference value was computed separately for each region by subtracting the sum of the time spent looking in the non-target quadrants from time spent looking in the target quadrant. Positive values indicate that the eye location was in the target quadrant more than combined time in the other quadrants and, thus, provide a conservative measure of quadrant preference for each region. Average preference values for non-direct participants were negative for the pool $(M=-67.93, S E M=18.68)$ and room $(M=-28.86, S E M=9.00)$. Average preference values for direct participants were positive for the pool $(M=100.39$, SEM $=21.94)$, and negative for the distal room $(M=-27.87, S E M=8.73)$. Direct comparisons of pool and room preference values were significant for direct participants (pool > room), $F(1$, $22)=33.08$, but were not significant for non-direct participants, $F(1,13)=3.59, p=.08$. This pattern indicates that direct participants focused more on the target quadrant in the pool than in the distal environment, whereas, the non-direct participants did not display a robust preference for the target quadrant in either region.

Another major pattern that emerged from Experiment 4 is that the eye location data within the pool corresponded well to the actual navigation data in direct participants, but not in non-direct participants. Although non-direct participants failed to discriminate among the quadrants of the pool on the basis of time spent in each quadrant, they did look at the target quadrant of the pool significantly more than each of the other regions. Like direct participants, non-direct participants looked in the SE quadrant, which was opposite the target quadrant, the least. Both the increased focus on the target quadrant and the lesser focus on the SE quadrant are likely due to the fact that 1) participants started in the S, or E during the probe and were, therefore always in close proximity to the SE quadrant and 2) all participants tended to look well in front of their actual position in the pool.

[Figure 12 about here.] 


\subsubsection{Summary}

During training, participants who learned to take direct trajectories the platform primarily looked at the distal room environment early during the trial, but looked primarily within the pool during the later aspects of trial. In contrast, participants who failed to take direct trajectories looked at distal cues transiently and focused primarily on the pool throughout the trial. During the no-platform probe trial, participants who learned to take direct trajectories primarily focused on the quadrant of the pool where the platform had been, but did not differentially focus on the various regions of the distal room. Further, unlike participants who failed to take direct trajectories during training, the pattern of navigation data during the probe trial for direct participants closely matched the corresponding eye location data obtained within the apparatus.

\section{General Discussion}

The results of the experiments reported support three basic conclusions regarding directional responding and place navigation and the role of room and apparatus cues in the control of these forms of navigation. First, the results of Experiment 1 establish that humans tested in a virtual Morris water task (VMWT) display a preference for directional responding over place navigation, as has been previously demonstrated in rats in the Morris water task (Hamilton et al., 2007, 2008a,b). Although there are obvious differences between real-world navigation by rats in the Morris water task and navigation by humans in the VMWT, comparable experimental design and test manipulations in each species produce very similar results in their respective tasks. Thus, the basic preference for directional responding in the Morris water task and related tasks holds considerable generality.

A second conclusion comes from the collective results of Experiments 1-3, which indicate that the pool wall is an important determinant of both the quality and type of navigation. When the pool is a prominent feature of the environment participants display a preference for directional responding (Experiment 1), and a similar result is observed when the pool is substantially reduced in size (Experiment 2). The latter manipulation was meant to approximate the reduction in pool wall height achieved by Hamilton et al. (2008b) when the pool is nearly filled with water, leaving only a small amount of the pool wall visible. Rats display a weak and transient preference for place navigation in this situation, therefore, we expected 
that humans may prefer place navigation in similar conditions. Our participants may not have shown a preference for place navigation because the pool, although reduced substantially in size, was easily detectable as a frame of reference and may have readily captured control of behavior. Hamilton et al. (2008a) suggested that the transient place navigation preference in the rat, which yields to a preference for directional responding with more extensive training, may have resulted from a change in the degree to which the apparatus controlled navigation as training continued. Weak apparatus control during the early stages of training would have allowed the distal room cues to exert more control and, therefore, support place navigation, whereas, increasing control by the pool could result in a shift to directional responding. It is important to consider that there are multiple potential explanations that could account for this pattern. For example, Hamilton et al. (2008b) suggested that the room cues provide direction information and the apparatus competes with room cues to provide distance information. When room cues are presented without apparatus cues, however, they provide both distance and direction information which allows them to control navigation to precise spatial locations in the room reference frame. Thus, directional responding may be preferred in any situation where the pool is detectable as a cue and can provide distance information and a source of competition, regardless of whether it is prominent cue or reduced as much as possible, as in Experiment 2. In Experiment 3, the VMWT was utilized to evaluate the effect of completely eliminating visual apparatus cues, a manipulation that cannot be achieved in a real-world experiment in the Morris water task. In this situation, participants showed a clear preference for place navigation, which, along with the results of Experiments 1 and 2, supports the conclusion that the presence of apparatus cues determine whether place navigation or directional responding is observed. These findings fit nicely with the previous data from rat studies and further question the generally accepted notion that navigation in the water task and related navigation tasks involves locating a goal based purely on its fixed spatial relationship to the available room cues.

Another curious observation from Experiments 1-3 is that the proportion of participants who learned to take direct trajectories to the platform increased as function of how much of the pool wall was visible. The highest proportion (70-75\%) was observed when the pool was a prominent feature, an intermediate proportion (about 50\%) took direct trajectories when the pool was reduced as much as possible, and the lowest (about 33\%) took direct 
trajectories when the pool was absent. This is consistent with the available data from the rat (Hamilton et al., 2008a,b) in which poorer learning was observed in rats trained with the pool wall reduced. Given that distal room cues are considered to be the primary determinant of behavior in spatial navigation tasks, it is interesting to consider that these results suggest that increasing the availability of distal room cues, as occurs whenever the pool is reduced, actually has deleterious effects on "spatial" learning. Conversely, increasing the ambiguous apparatus cues actually enhances direct navigation that is typically characteristic of place navigation. At present we cannot offer an explanation of these observations, however, it is hoped that future computational modeling efforts can identify the properties of room and apparatus cue control that contribute to these effects. Most importantly, these observations along with the results of the test trials described above, firmly establish the importance of apparatus cues in the control of navigation.

The third conclusion comes from the eyetracking data obtained in Experiment 4 . The results indicate that participants who master the VMWT tend to look at the distal room cues early during a trial, but switch to focusing on the pool as the trial progresses. In contrast, participants who did not learn to take direct trajectories did not show this lawful pattern of sequential stimulus control. Further, participants who learned to navigate to the platform spent a considerable proportion of their time looking at the location of the platform in the pool during a no-platform probe trial. This is of some importance, when one considers that the pool, which is ambiguous with respect to spatial locations, is nonetheless the primary region on which participants focus as they navigate. These observations support the movement vector hypothesis of Hamilton et al. (2007) in that the directionality of the initial trajectory with respect to the distal room cues would need to be established early, and the pool would subsequently provide distance information.

Like Experiment 3, Experiment 4 represents the use of virtual navigation technology to obtain data and address questions that are difficult or impossible to address in rodent experiments, in this case, dynamic estimation of precisely where participants were looking as they navigated to the goal. The potential uses of this technology to address basic questions regarding stimulus control in navigation represent an important and exciting development in the area of navigation research. We also believe that the combination of eyetracking and navigation data can provide a potentially rich methodological framework for developing and 
evaluating computational models of navigation.

Computational modeling of navigation is a diverse and rich area of research that has been extensively applied to address basic processes involved in animal navigation and their neurobiological bases. A thorough review of the literature on modeling of spatial navigation is far beyond the scope of the present paper, however, we wish to draw attention to two implications of the present results and the present methodology for computational approaches.

The results of the rodent data described above and the novel human data reported here question some of the most basic assumptions about what behaviors need to be quantified and modeled and what stimulus information is required for modeling the behavior of interest. All computational models must address and make assumptions about the inputs, intermediate processing, and outputs. The present findings in humans and the extant literature on rat performance in the Morris water task suggests that both distal room cues and pool cues should be represented as inputs to any computational model that attempts to accurately model navigation in the water task and related methods. A common approach is to provide input from distal cues in the form of distances from individual cues or related metrics such as subtended visual angle (see e.g., Schmajuk (1997); Sharp (1991); Sharp et al. (1996); Wilkie and Palfrey (1987)), whereas no information regarding the apparatus other than its role as a boundary that cannot be crossed are provided. Given that most researchers have generally agreed that navigation in the water task involves learning the location of the platform based on its fixed spatial relationship to a constellation of distal cues, these types of inputs are reasonable given the assumption that distances to the available room cues are critical. Of course, if navigation in the water task were only a matter of learning the distances to several distal cues from the release point we should expect to observe place navigation rather than directional responding. In short, the evidence for directional responding suggests that input from distal cues is either not of this type, or if it is, it does not result in the type of control that is assumed when distance information is used as the primary input information. Both distal cue and apparatus cue information are critical for accurately modeling navigation in the water task and, therefore, future computational approaches designed to model navigation in the water task should utilize orientation/directional information based on distal cues and distance information from the apparatus cues as input signals.

The types of distance or visual angle inputs described above are also related to assumptions 
regarding the behavioral processes involved in navigation. For example, Wilkie and Palfrey (1987) presented a perceptual matching model in which the model forms a perceptual memory of the visual appearance of several distal cues at the goal site. In this model, navigation from a release point to the goal involves reducing the error between the current view and the perceptual memory associated with the goal location. This assumes that navigation involves recognizing spatial locations and navigating to a precise location based on its fixed spatial relationship to the available cues. Thus, such an approach would predict place navigation rather than directional responding. In fairness to Wilkie and Palfrey (1987), they assumed that the apparatus was not a critical determinant of spatial navigation. This is common in the behavioral and computational literature, likely because the apparatus in the water task is assumed to provide no information capable of disambiguating spatial locations. Our results suggest that navigation in the water task does not depend upon singular processes such as perceptual matching or place recognition, but rather involves distinct and sequential processes related to trajectory selection and distance computation.

Recently, Cheng and colleagues (Cheng, 2008; Cheung et al., 2008) have suggested that view-based matching, rather than place matching, may account for directional responding. Under this view, the critical process to be modeled is not one of recognizing a particular place relative to distal cues alone, but rather matching the perceived view of salient features of the environment, as viewed from the platform, which would include the pool wall. When the pool is repositioned during our shift tests, the relative position in the pool would most closely match the view from the platform location used during training compared to any other location in the pool, although some generalization decrement may be observed. View-based matching can account for a great deal of data obtained in the rodent and human experiments, particularly the basic findings on the preference for directional responding over place navigation. It is not clear, however, how such a approach would account for the dynamic changes in stimulus control during an individual trial and there are some data from our previous experiments that present a problem for this approach. Hamilton et al. (2007) conducted an experiment in which the platform was marked by a conspicuous cue during training. During a test trial, the pool was repositioned and the cued platform was either placed in the absolute location in the room or in the relative location in the pool. Surprisingly, rats tested with the platform in the absolute location did poorly and either navigated in the opposite direction from the platform 
(toward the relative location) or were otherwise disrupted, whereas rats tested with the cued platform in the relative location swam directly to the platform. Although not designed as a test for the view-based matching hypothesis, it seems reasonable to expect that the cue above the platform would be a salient feature of the view while navigating to the platform, yet rats tested with the platform in the absolute location often swam in the direction of the platform rather than to the cue that marked the platform. This suggests that the basic processes involved in selecting a trajectory are independent of subsequent processes such as navigating on the basis of a single cue that marks the goal (see also, Hamilton et al. (2004)).

Given the sequential nature of control postulated by our movement vector hypothesis, dynamic modeling of moment to moment spatial navigation will be critical for capturing the basic features of navigation involved in directional responding. Trial level models that attempt to capture molar features of behavior or models that postulate singular behavioral processes will not be sufficient to capture the behaviors of interest. Thus, it seems that there are good reasons to consider dynamic changes in behaviors and controlling processes in modeling approaches, and to undertake systematic comparisons of these approaches with more traditional single process and molar approaches. We are currently evaluating several approaches to this particular issue in our laboratory, but we hope that other investigators will pursue this problem as well.

The foregoing discussion has emphasized the importance of considering the multiple, dynamic changes in stimulus control and associated behaviors that are involved in navigation tasks. We conclude with a discussion of the potential benefits of using the eyetracking technology described here in the service of developing and evaluating computational models. In addition to potential constraints the information from the basic behavioral observations described here can provide, the methods employed in Experiment 4 may also provide an important source of input and desired output data. Computational models of rat navigation must make fundamental assumptions about primary and higher order visual processes, in part because the precise stimulus information at any particular point in time is now known. One potential benefit of using eyetracking technology in virtual navigation is that both the $2 \mathrm{~d}$ environmental display and the precise location of the gaze on the display and in the $3 \mathrm{~d}$ environment can be obtained for each sample during a navigation trial. The precise behavioral output in terms of eye movements and navigation (forward movement and rotation) can be obtained simultane- 
ously. Thus, one approach to modeling the processes of interest would be to use the moment to moment views of the environment (as shown on the display) and related eye locations as inputs into the model, thereby eliminating the need to make assumptions regarding the type of information provided by the available cues. Further, the behavioral outputs are also be known and can be used to train models to reproduce human data in the VMWT in the service of developing dynamic models that capture the changes in control by room and apparatus cues. If successful, this approach may prove invaluable in developing and evaluating models of navigation. Given the basic similarities in rodent and human navigation noted here, such models may generalize to other domains, such as models designed to capture the physiological processes involved in mammalian navigation.

\section{References}

Akers, K. G., Candelaria, F. T., Hamilton, D. A., 2007. Preweanling rats solve the Morris water task via directional navigation. Behav. Neurosci. 121, 1426-1430.

Astur, R. S., Ortiz, M., Sutherland, R. J., 1998. A characterization of performance by men and women in a virtual Morris water task. Behav. Brain Res. 93, 185-190.

Blodgett, H. C., McCutchan, K., Mathews, R., 1949. Spatial learning in the T-maze: The influence of direction, turn, and food location. J. Exp. Psychol. 39, 800-809.

Brandt, T., Schautzer, F., Hamilton, D. A., Bruning, R., Markowitsch, H. J., Kalla, R., Darlington, C., Smith, P., Strupp, M., 2005. Vestibular loss causes hippocampal atrophy and impaired spatial memory in humans. Brain 128, 2732-2741.

Chamizo, V. D., Aznar-Casanova, J. A., Artigas, A. A., 2003. Human overshadowing in a virtual pool: Simple guidance is a good competitor against locale learning. Learn. Motiv. 34, 262-281.

Cheng, K., 2008. Whither geometry?:Troubles of the geometric module. Trends Cog. Sci. 12, 355-361.

Cheung, A., Stuerzl, W., Zeil, J., Cheng, K., 2008. The information content of panoramic images ii: View-based navigation in nonrectangular experimental arenas. J. Exp. Psychol.: Anim. Behav. Process. 34, $15-30$. 
Deutsch, J. A., 1960. The structural basis of behavior. University of Chicago Press, Chicago, IL.

Driscoll, I., Hamilton, D. A., Petropoulos, H., Yeo, R. A., Brooks, W. M., Baumgartner, R. N., Sutherland, R. J., 2003. The aging hippocampus: cognitive, biochemical and structural findings. Cerebral Cortex 13, 1344-1351.

Driscoll, I., Hamilton, D. A., Yeo, R. A., Brooks, W. M., Sutherland, R. J., 2005. Virtual navigation in humans: the impact of age, sex, and hormones on place learning. Hormones Behav. 47, 326-335.

Gallagher, M., Burwell, R., Burchinal, M., 1993. Severity of spatial-learning impairment in aging - development of a learning index for performance in the Morris water maze. Behav. Neurosci. 107, 618-626.

Hamilton, D. A., Akers, K. G., Johnson, T. E., Rice, J. P., Candelaria, F. T., , Redhead, E. S., 2008a. Evidence for a shift from place navigation to directional responding in one variant of the morris water task. J. Exp. Psychol.: Anim. Behav. Process. in press.

Hamilton, D. A., Akers, K. G., Johnson, T. E., Rice, J. P., Candelaria, F. T., Sutherland, R. J., Weisend, M. P., Redhead, E. S., 2008b. The relative influence of place and direction in the Morris water task. J. Exp. Psychol.: Anim. Behav. Process. 34, 31-53.

Hamilton, D. A., Akers, K. G., Weisend, M. P., Sutherland, R. J., 2007. How do room and apparatus cues control navigation in the Morris water task?: Evidence for distinct contributions to a movement vector. J. Exp. Psychol.: Anim. Behav. Process. 33, 100-114.

Hamilton, D. A., Driscoll, I., Sutherland, R. J., 2002. Human place learning in a virtual Morris water task: Some important constraints on the flexibility of place navigation. Behav. Brain Res. 129, 159-170.

Hamilton, D. A., Kodituwakku, P., Sutherland, R. J., Savage, D. D., 2003. Children with Fetal Alcohol Syndrome are impaired at place learning but not cued-navigation in a virtual Morris water task. Behav. Brain Res. 143, 85-94.

Hamilton, D. A., Rosenfelt, C. S., Whishaw, I. Q., 2004. Sequential control of navigation by locale and taxon cues in the Morris water task. Behav. Brain Res. 154, 385-397. 
Hamilton, D. A., Sutherland, R. J., 1999. Blocking in human place learning: Evidence from virtual navigation. Psychobiol. 27, 453-461.

Hanlon, F. M., Weisend, M. P., Hamilton, D. A., Jones, A., Thoma, R. J., Huang, M.-X., Martin, K., Yeo, R. A., Miller, G. A., nive, J. M. C., 2006. Impairment on the hippocampaldependent virtual Morris water task in schizophrenia. Schiz. Res. 87, 67-80.

Horne, M. R., Martin, G. M., Harley, C. W., Skinner, D. M., 2007. Where am i? distal cue use requires sensitivity to start location change in the rat. J. Exp. Psychol.: Anim. Behav. Process. 33, 92-99.

Hufner, K., Hamilton, D. A., Kalla, R., Stephan, T., Glasauer, S., Ma, J., Bruning, R., Markowitsch, H. J., Labudda, K., Schichor, C., Strupp, M., Brandt, T., 2007. Spatial memory and hippocampal volume in humans with unilateral vestibular deafferentation. Hippocampus. 17, $471-485$.

Hull, C. L., 1934a. The concept of the habit-family hierarchy and maze learning: Part I. Psychol. Rev. 41, 33-54.

Hull, C. L., 1934b. The concept of the habit-family hierarchy and maze learning: Part II. Psychol. Rev. 41, 134-152.

Hull, C. L., 1943. Principles of Behavior: An Introduction to Behavior Theory. AppletonCentury-Crofts, New York.

Jacobs, W. J., Thomas, K. G. F., Laurance, H. E., Nadel, L., 1998. Place learning in virtual space ii. topographical relations as one dimension of stimulus control. Learn. Motiv. 29, $288-308$.

Knierim, J. J., Rao, G., 2003. Distal landmarks and hippocampal place cells: Effects of relative translation versus rotation. Hippocampus 13, $604-617$.

Moffat, S. D., Hampson, E., Hatzipantelis, M., 1998. Navigation in a "virtual" maze: Sex differences and correlation with psychometric measures of spatial ability in humans. Evol. Human Beh. 19, 73-87.

Morris, R. G. M., 1981. Spatial localization does not require the presence of local cues. Learn. Motiv. 12, 239-260. 
Morris, R. G. M., 1984. Developments of a water-maze procedure for studying spatial-learning in the rat. J. Neurosci. Methods. 11, 47-60.

O'Keefe, J., Nadel, L., 1978. The Hippocampus as a cognitive map. Clarendon Press, Oxford, England.

Olton, D. S., Becker, J. T., Handelmann, G. E., 1979. Hippocampus, space, and memory. Behav. Brain Sci. 2, 313 - 322.

Redhead, E. S., Hamilton, D. A., 2007. Interaction between locale and taxon strategies in human spatial learning. Learn. Motiv. 38, 262-283.

Sandstrom, N. J., Kaufman, J., Huettel, S. A., 1998. Males and females use different distal cues in a virtual environment navigation task. Cog. Brain Res. 6, 351-360.

Schautzer, F., Hamilton, D., Kalla, R., Strupp, M., Brandt, T., Oct 2003. Spatial memory deficits in patients with chronic bilateral vestibular failure. Ann. NY Acad. Sci. 1004, 316324 .

Schmajuk, N. A., 1997. Animal learning and cognition: A neural network approach. Cambridge University Press, Cambridge, UK.

Sharp, P. E., 1991. Computer simulation of hippocampal place cells. Psychobiol. 19, 103-115.

Sharp, P. E., Blair, H. T., Brown, M., 1996. Neural network modeling of the hippocampal formation spatial signals and their possible role in navigation: A modular approach. Hippocampus. $6,720-734$.

Skinner, D. M., Etchegary, C. M., Ekert-Maret, E. C., Baker, C. J., Harley, C. W., Evans, J. H., Martin, G. M., 2003. An analysis of response, direction, and place learning in an open field and T maze. J. Exp. Psychol.: Anim. Behav. Process. 29, 3 - 13.

Stringer, K. G., Martin, G. M., Skinner, D. A., 2005. The effects of hippocampal lesions on response, direction, and place learning in rats. Behav. Neurosci. 119, 946 - 952.

Sutherland, R. J., Dyck, R. H., 1984. Place navigation by rats in a swimming pool. Can. J. Psychol. 38, 322-347. 
Sutherland, R. J., Hamilton, D. A., 2004. Rodent spatial navigation: At the crossroads of cognition and movement. Neurosci. Biobehav. Rev. 28, 687-697.

Tolman, E. C., 1948. Cognitive maps in rats and men. Psychol. Rev. 55, 189-208.

Tolman, E. C., Ritchie, B. F., Kalish, D., 1946. Studies in spatial learning. II. place learning versus response learning. J. Exp. Psychol. 3, 221 - 229.

Wilkie, D. M., Palfrey, R., 1987. A computer simulation model of rats' place navigation in the Morris water maze. Behav. Res. Methods Instrum. Comput. 19, 400-403.

Yoganarasimha, D., Knierim, J. J., 2005. Coupling between place cells and head direction cells during relative translations and rotations of distal landmarks. Exp. Brain Res. 160, 344 359. 


\section{List of Figures}

1 Layout of the testing room used by Hamilton et al. (2007, 2008a,b) showing the room geometry and location of prominent visual cues (gray or black rectangles). The pool was located in one of two positions that were separated by $75 \mathrm{~cm}$ (the pool radius). The escape platform was always placed at locations $\mathrm{B}$, which represents the same absolute spatial location within the room reference frame for both pool positions. Locations $\mathrm{A}$ and $\mathrm{C}$ represent comparison locations that are in the opposite quadrant from the platform location (B) for pool positions 1 and 2, respectively. For the Shift groups these locations correspond to the relative location of the platform within the pool. The dark circles inside the pool mark the four release points used during hidden platform training and the squares represent the two release points sampled for the no platform probe trial. 37

2 A) Layout of the virtual environment showing the location of distal room cues and pool locations. The distal room walls are laid flat. The layout is to scale; Units of arbitrary length used to describe the environment in the General Method section are illustrated by the black bar. Pool positions A and B are represented by the black and grey circles, respectively. B) Example view of the environment showing the pool and distal room cues from a participant's perspective. . . . . . . . . . . . . . . . . . . .

3 Representative probe trial swim paths for participants from the Shift and No Shift groups of Experiment 1. Paths were selected from participants who learned to take direct trajectories during training and who had median latencies to enter the preferred region during the probe trial (absolute for the No Shift group and relative for the Shift group). The large, thin circle shown for the Shift participant indicates the pool position used during training. The thick circles indicate the pool position during the probe trial. The light gray circles within the pool mark the two critical regions (1 unit diameter) around the absolute (dark gray square), relative (light gray square) or opposite location (open square) used for analysis. The initial trajectory, defined as the path taken from the release point until one of the two critical circular regions was entered, is shown in filled black circles. The remainder of the path for the probe trial is shown as a thin black line.

4 Probe trial dependent measures (Mean + SEM) for each group. A) Latency to enter the 1 unit diameter circular region around the two locations of interest. B) Mean distance ("proximity") from the two critical locations. C) Number of times each critical location was crossed. D) Time spent in each of the two critical circular regions. . . . . . . . . . . . . . . . . . . 
5 Representative probe trial swim paths for participants from the Shift and No Shift groups of Experiment 2. Paths were selected from participants who learned to take direct trajectories during training and who had median latencies to enter the preferred region during the probe trial (absolute for the No Shift group and relative for the Shift group). The large, thin circle shown for the Shift participant indicates the pool position used during training. The thick circles indicate the pool position during the probe trial. The light gray circles within the pool mark the two critical regions (1 unit diameter) around the absolute (dark gray square), relative (light gray square) or opposite location (open square) used for analysis. The initial trajectory, defined as the path taken from the release point until one of the two critical circular regions was entered, is shown in filled black circles. The remainder of the path for the probe trial is shown as a thin black line.

Probe trial dependent measures (Mean + SEM) for each group. A) Latency to enter the 1 unit diameter circular region around the two locations of interest. B) Mean distance ("proximity") from the two critical locations. C) Number of times each critical location was crossed. D) Time spent in each of the two critical circular regions. . . . . . . . . . . . . . . . . .

7 Representative probe trial swim paths for participants from the Shift and No Shift groups of Experiment 2. Paths were selected from participants who learned to take direct trajectories during training and who had median latencies to enter the preferred region during the probe trial (absolute for the No Shift group and relative for the Shift group). The large, thin circle shown for the Shift participant indicates the pool position used during training. The thick circles indicate the pool position during the probe trial. The light gray circles within the pool mark the two critical regions (1 unit diameter) around the absolute (dark gray square), relative (light gray square) or opposite location (open square) used for analysis. The initial trajectory, defined as the path taken from the release point until one of the two critical circular regions was entered, is shown in filled black circles. The remainder of the path for the probe trial is shown as a thin black line.

8 Probe trial dependent measures (Mean + SEM) for each group. A) Latency to enter the 1 unit diameter circular region around the two locations of interest. B) Mean distance ("proximity") from the two critical locations. C) Number of times each critical location was crossed. D) Time spent in each of the two critical circular regions. . . . . . . . . . . . . . . . . . . .

9 Representative ethograms during the final four training trials for one direct (left) and one non-direct (right) participant showing the region of the environment (4 quadrants in the pool and in the distal room) where eye fixations were located as a function of time (left to right). The platform was located in the NW quadrant of the pool. The light and dark grey alternating background is only included to help discriminate between regions and does not provide any information about the eye location. Black sections indicate the region where the eye location was measured. Gaps at the beginning of the ethogram indicate that the first movement in the trial had not yet been made. Gaps also appear rarely when participants looked at the boundary between two regions. . . . . 
10 Mean percentage (and SEM) of time participants classified as direct (A) or non-direct (B) spent looking at the distal room environment or within the pool during the final block of hidden platform training as a function of the trial segment (.05 represents 0-0.5, .10 represent .06 - .10, etc. . . . . . . . . . .

11 Representative ethogram (left) and dwell plot (right) during the no-platform probe trial for participants classified as direct or non-direct. The ethograms represent $60 \mathrm{sec}$ of navigation and follow the same conventions described in Fig. 9. The dwell plots were created by taking individual regions in the room on a grid comprised $.25 \mathrm{X} .25$ unit sections. The center of the red circles represents the location of the eye position in 3-d space and the diameter of the circle represents the total amount of time during the probe trial the eyes were positioned at that location. Because the duration of the trial was identical for both participants shown here, the relative diameters of the circles can be directly compared. The large circle shown for the direct participant is located in the target quadrant

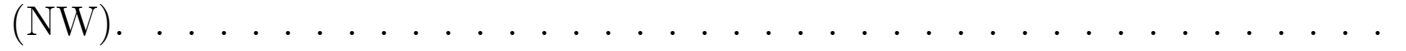

12 A) Mean percentage $(+\mathrm{SEM})$ time that direct and non-direct participants spent in each quadrant of the pool during the no-platform probe trial. B) Mean percentage $(+$ SEM) time that direct and non-direct participants spent looking in each of 8 regions of environment (4 quadrants in the pool and 4 quadrants in the distal room). The platform was located in the NW quadrant of the pool during training. 
Figure 1.

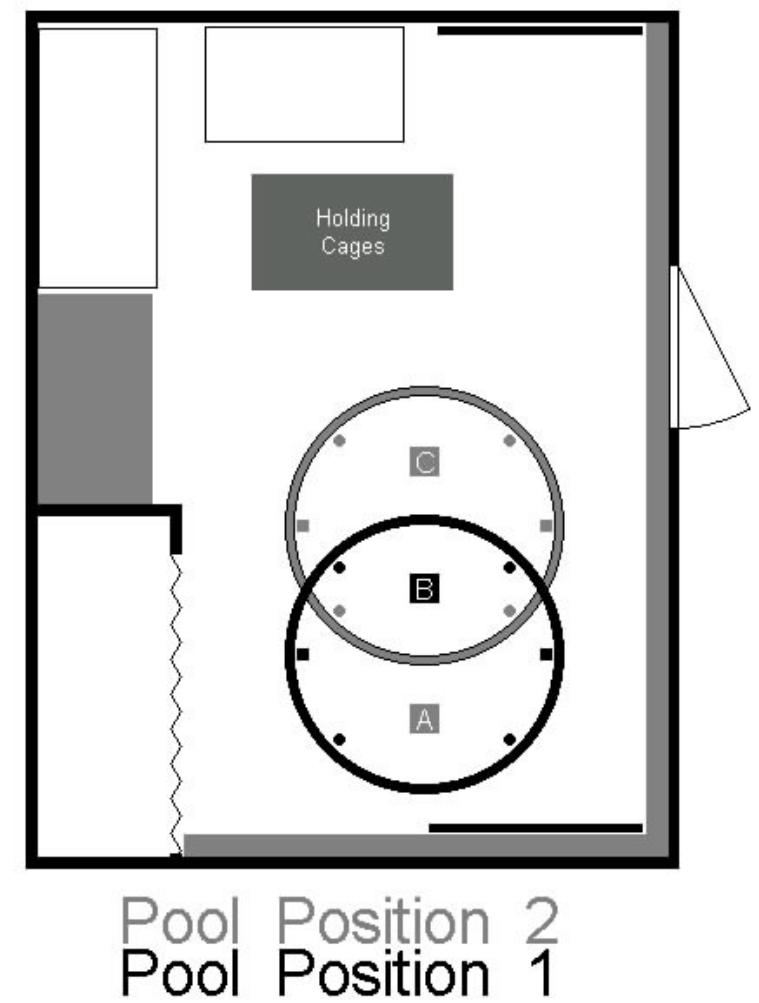

North $\longleftarrow$

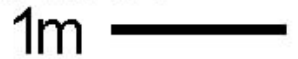

Figure 1: Layout of the testing room used by Hamilton et al. (2007, 2008a,b) showing the room geometry and location of prominent visual cues (gray or black rectangles). The pool was located in one of two positions that were separated by $75 \mathrm{~cm}$ (the pool radius). The escape platform was always placed at locations B, which represents the same absolute spatial location within the room reference frame for both pool positions. Locations $\mathrm{A}$ and $\mathrm{C}$ represent comparison locations that are in the opposite quadrant from the platform location (B) for pool positions 1 and 2, respectively. For the Shift groups these locations correspond to the relative location of the platform within the pool. The dark circles inside the pool mark the four release points used during hidden platform training and the squares represent the two release points sampled for the no platform probe trial. 
Figure 2.
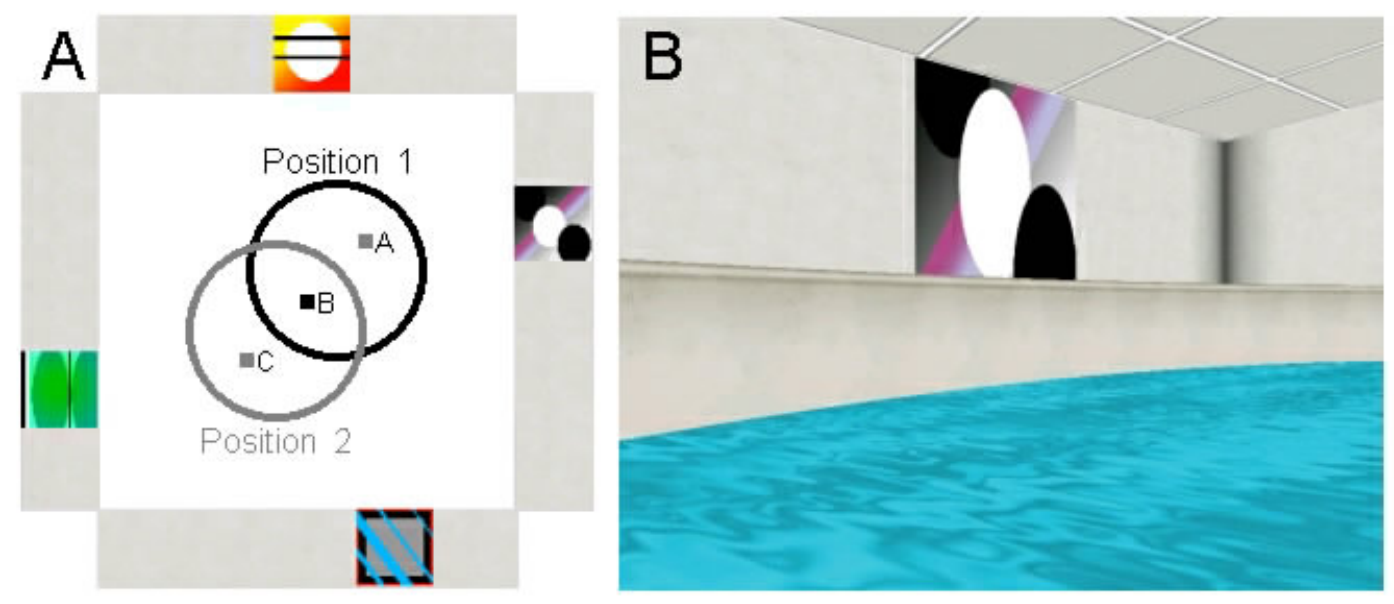

Figure 2: A) Layout of the virtual environment showing the location of distal room cues and pool locations. The distal room walls are laid flat. The layout is to scale; Units of arbitrary length used to describe the environment in the General Method section are illustrated by the black bar. Pool positions A and B are represented by the black and grey circles, respectively. B) Example view of the environment showing the pool and distal room cues from a participant's perspective. 
Figure 3.
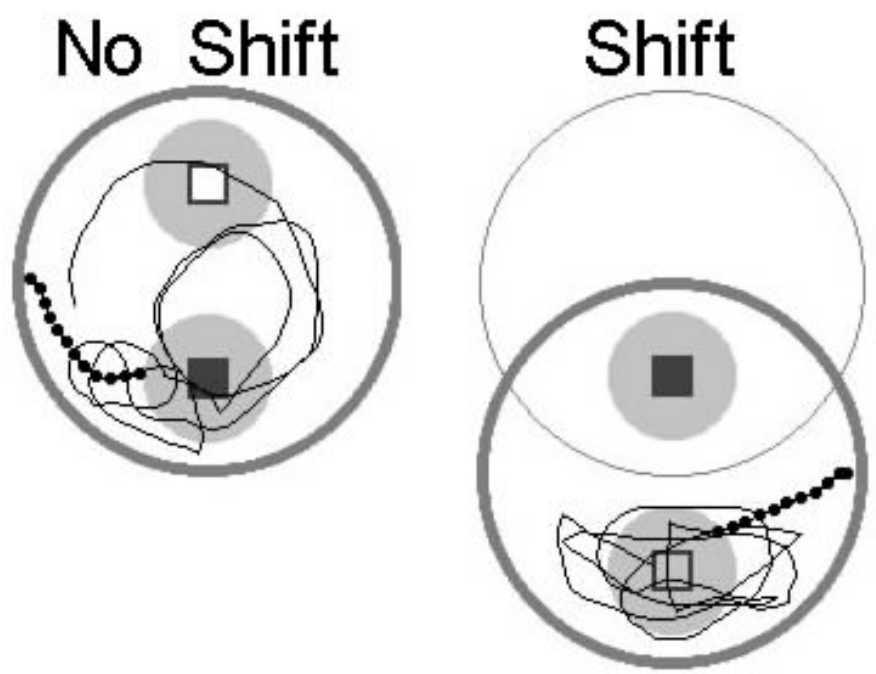

\section{Absolute $\square$ Relative $\square$ Opposite location location location}

Figure 3: Representative probe trial swim paths for participants from the Shift and No Shift groups of Experiment 1. Paths were selected from participants who learned to take direct trajectories during training and who had median latencies to enter the preferred region during the probe trial (absolute for the No Shift group and relative for the Shift group). The large, thin circle shown for the Shift participant indicates the pool position used during training. The thick circles indicate the pool position during the probe trial. The light gray circles within the pool mark the two critical regions (1 unit diameter) around the absolute (dark gray square), relative (light gray square) or opposite location (open square) used for analysis. The initial trajectory, defined as the path taken from the release point until one of the two critical circular regions was entered, is shown in filled black circles. The remainder of the path for the probe trial is shown as a thin black line. 
Figure 4.
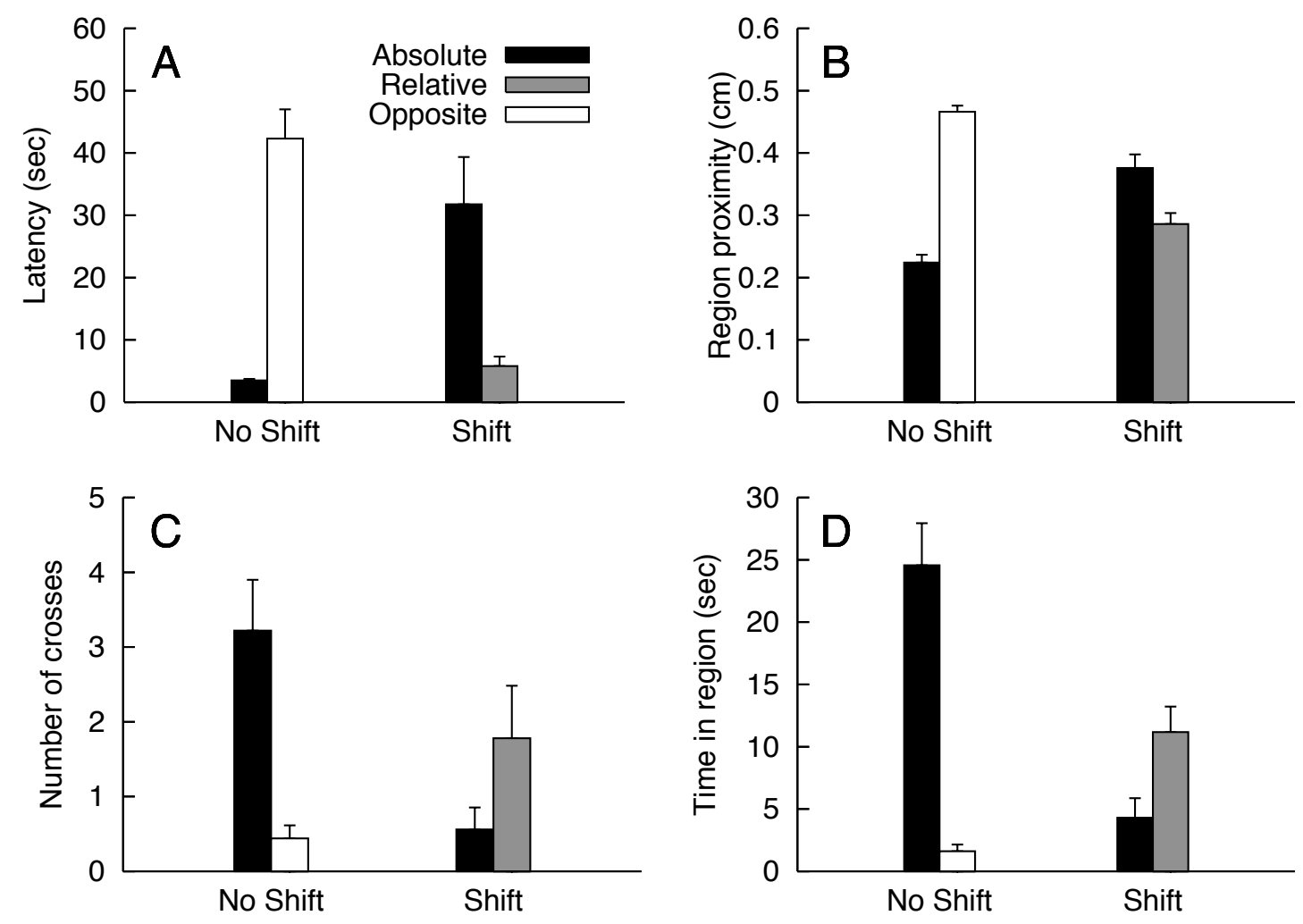

Figure 4: Probe trial dependent measures (Mean + SEM) for each group. A) Latency to enter the 1 unit diameter circular region around the two locations of interest. B) Mean distance ("proximity") from the two critical locations. C) Number of times each critical location was crossed. D) Time spent in each of the two critical circular regions. 
Figure 5 .
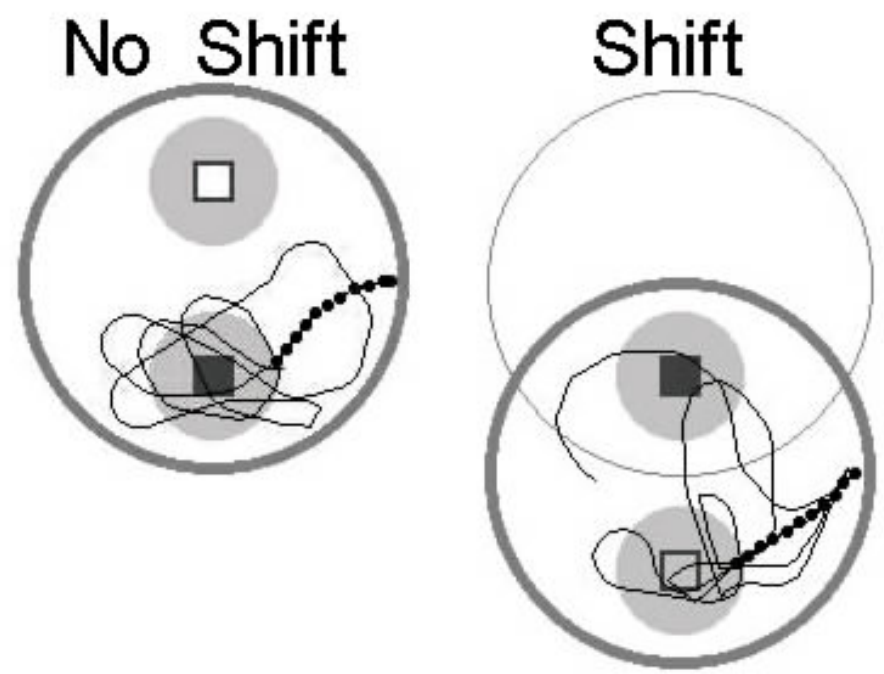

\section{Absolute $\square$ Relative $\square$ Opposite location location location}

Figure 5: Representative probe trial swim paths for participants from the Shift and No Shift groups of Experiment 2. Paths were selected from participants who learned to take direct trajectories during training and who had median latencies to enter the preferred region during the probe trial (absolute for the No Shift group and relative for the Shift group). The large, thin circle shown for the Shift participant indicates the pool position used during training. The thick circles indicate the pool position during the probe trial. The light gray circles within the pool mark the two critical regions (1 unit diameter) around the absolute (dark gray square), relative (light gray square) or opposite location (open square) used for analysis. The initial trajectory, defined as the path taken from the release point until one of the two critical circular regions was entered, is shown in filled black circles. The remainder of the path for the probe trial is shown as a thin black line. 
Figure 6.
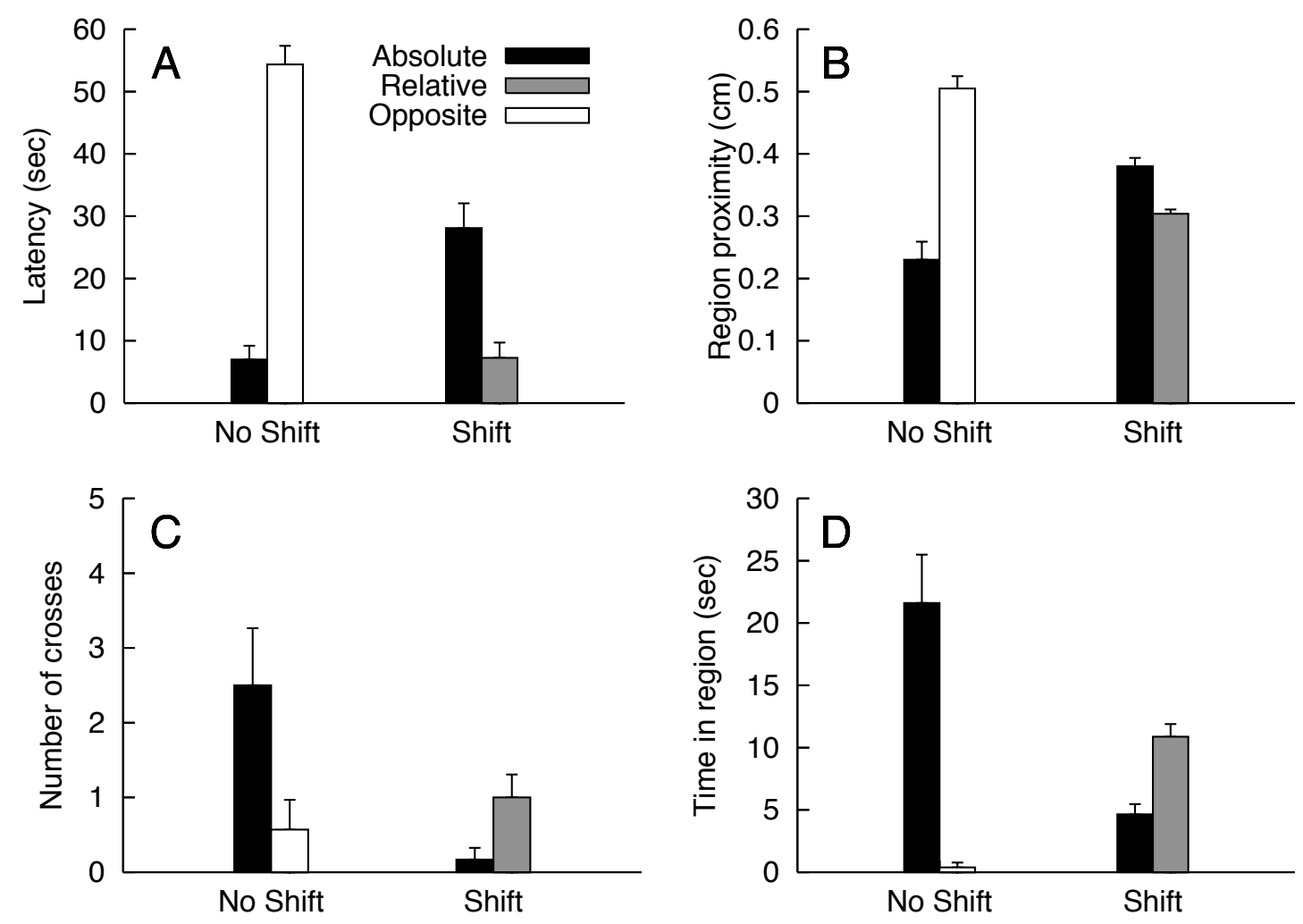

Figure 6: Probe trial dependent measures (Mean + SEM) for each group. A) Latency to enter the 1 unit diameter circular region around the two locations of interest. B) Mean distance ("proximity") from the two critical locations. C) Number of times each critical location was crossed. D) Time spent in each of the two critical circular regions. 
Figure 7.
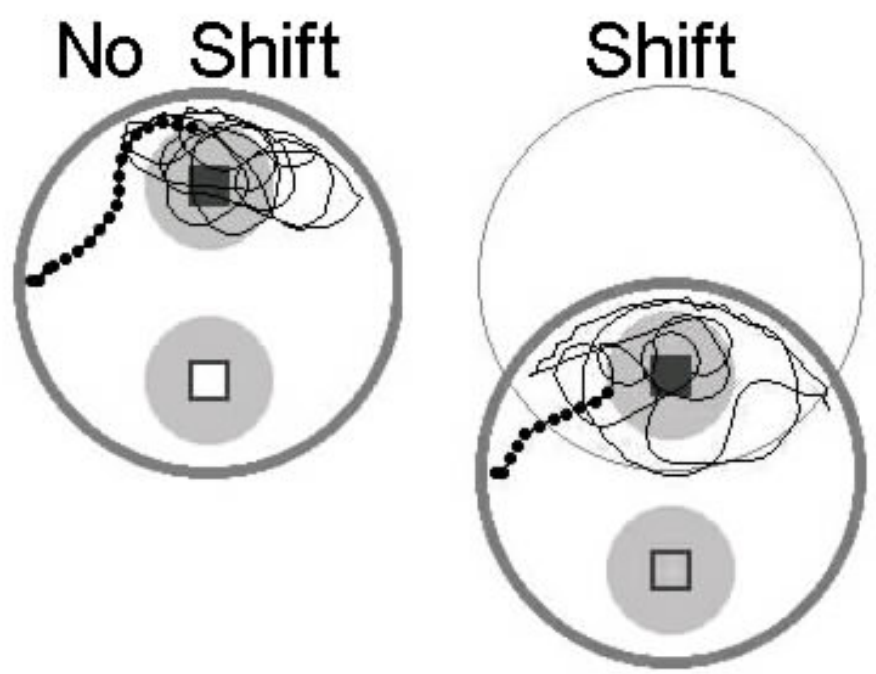

\section{Absolute $\square$ Relative $\square$ Opposite location location location}

Figure 7: Representative probe trial swim paths for participants from the Shift and No Shift groups of Experiment 2. Paths were selected from participants who learned to take direct trajectories during training and who had median latencies to enter the preferred region during the probe trial (absolute for the No Shift group and relative for the Shift group). The large, thin circle shown for the Shift participant indicates the pool position used during training. The thick circles indicate the pool position during the probe trial. The light gray circles within the pool mark the two critical regions (1 unit diameter) around the absolute (dark gray square), relative (light gray square) or opposite location (open square) used for analysis. The initial trajectory, defined as the path taken from the release point until one of the two critical circular regions was entered, is shown in filled black circles. The remainder of the path for the probe trial is shown as a thin black line. 
Figure 8.
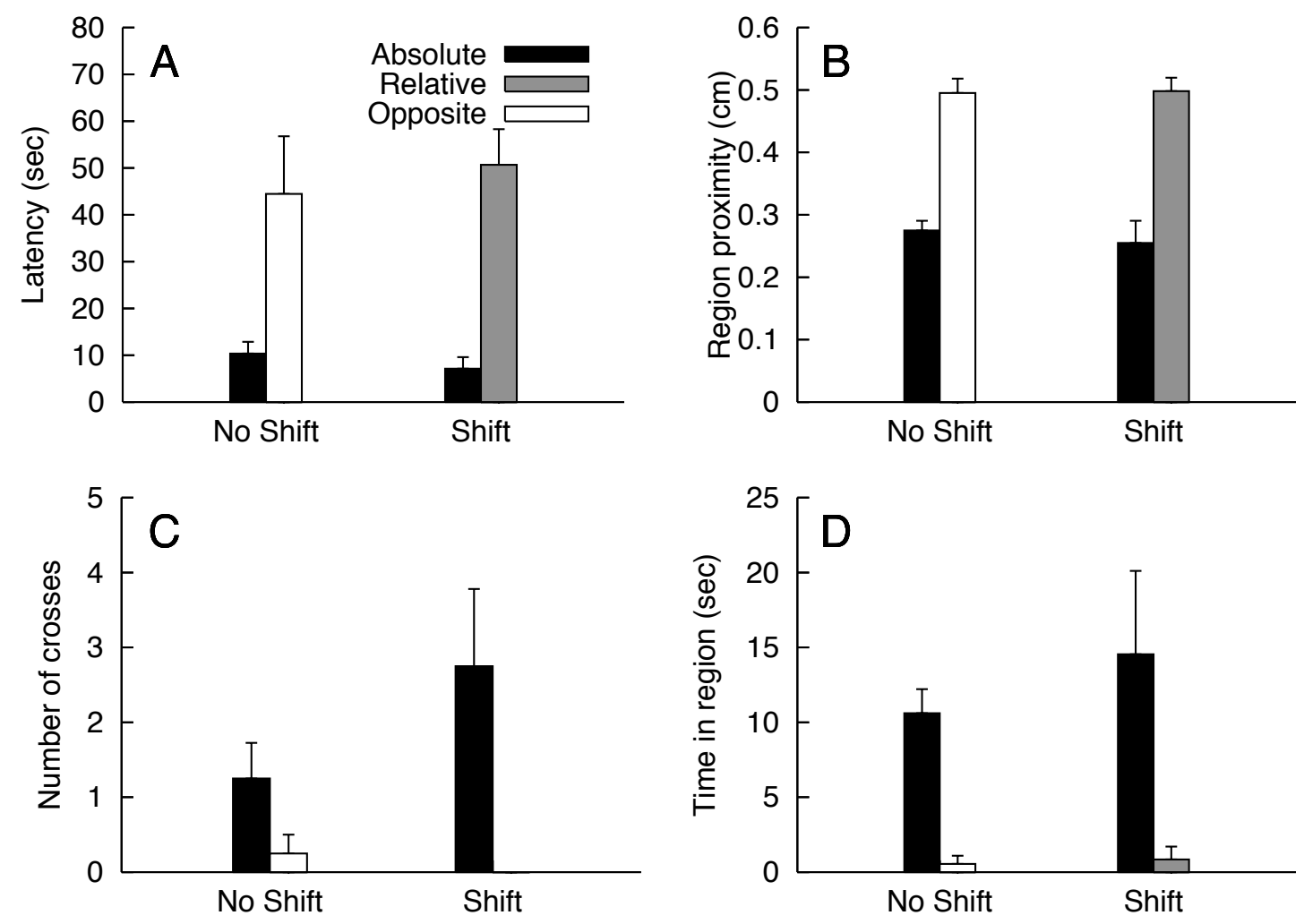

Figure 8: Probe trial dependent measures (Mean + SEM) for each group. A) Latency to enter the 1 unit diameter circular region around the two locations of interest. B) Mean distance ("proximity") from the two critical locations. C) Number of times each critical location was crossed. D) Time spent in each of the two critical circular regions. 
Figure 9.

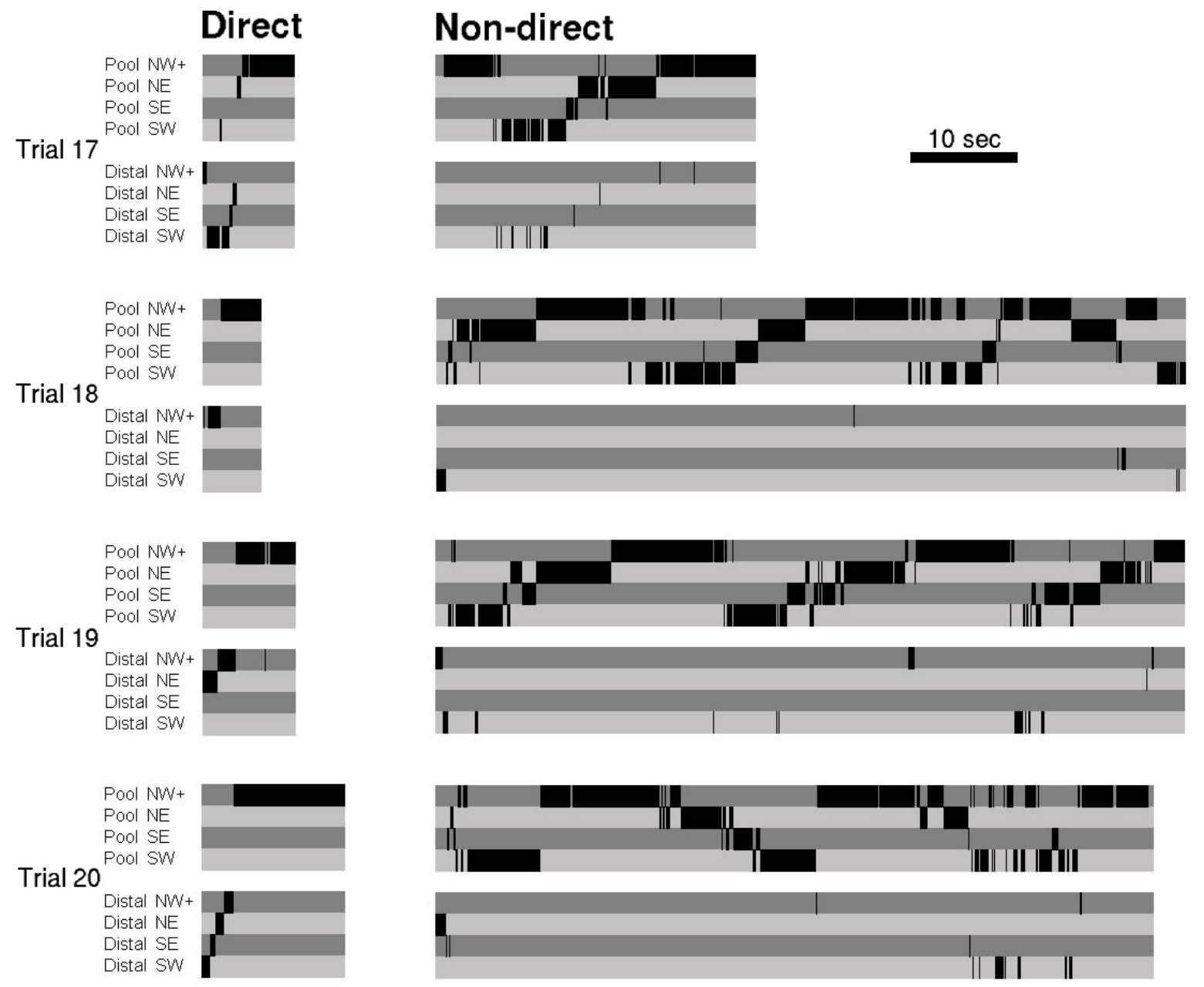

Figure 9: Representative ethograms during the final four training trials for one direct (left) and one non-direct (right) participant showing the region of the environment (4 quadrants in the pool and in the distal room) where eye fixations were located as a function of time (left to right). The platform was located in the NW quadrant of the pool. The light and dark grey alternating background is only included to help discriminate between regions and does not provide any information about the eye location. Black sections indicate the region where the eye location was measured. Gaps at the beginning of the ethogram indicate that the first movement in the trial had not yet been made. Gaps also appear rarely when participants looked at the boundary between two regions. 
Figure 10.
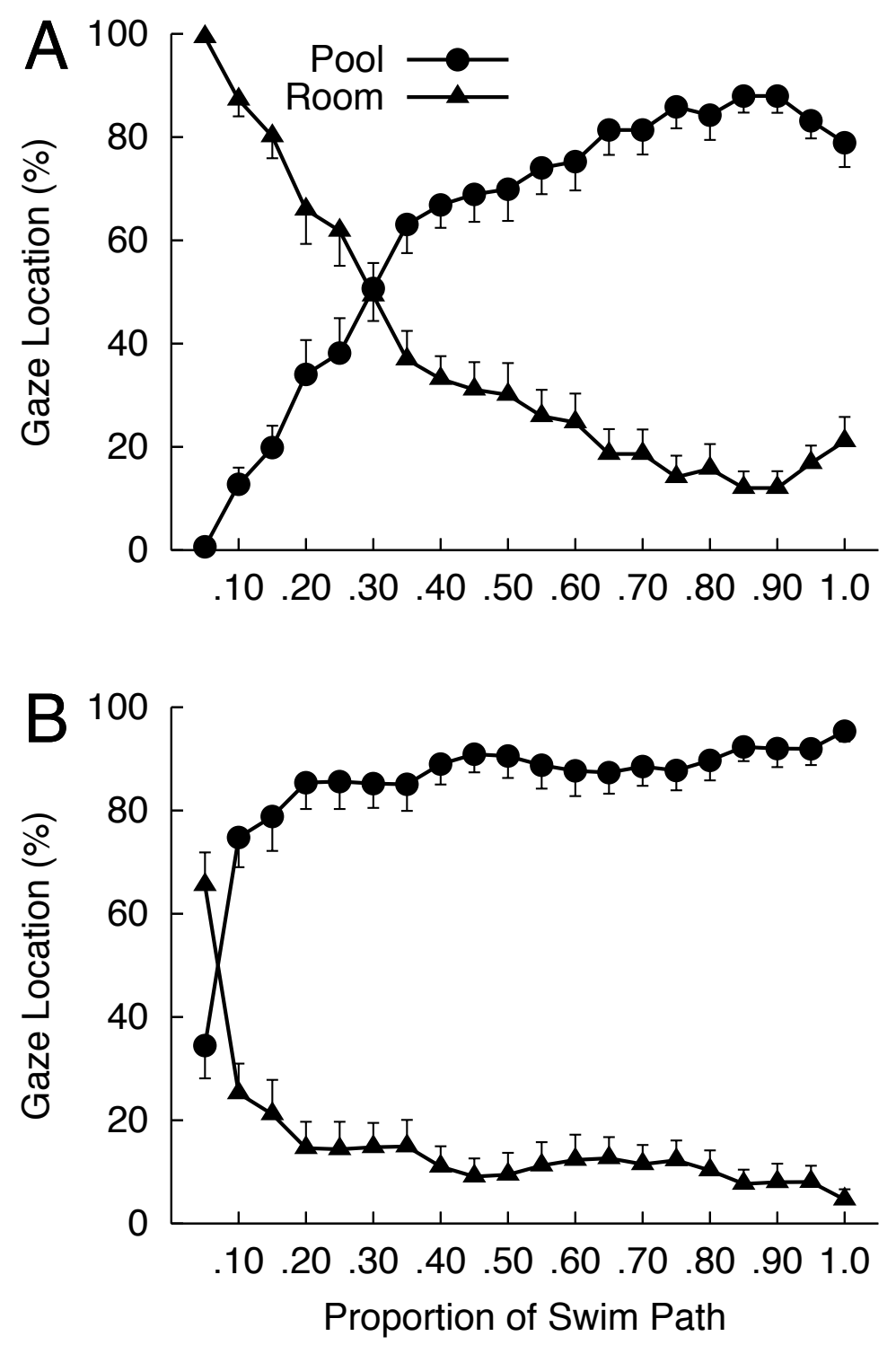

Figure 10: Mean percentage (and SEM) of time participants classified as direct (A) or non-direct (B) spent looking at the distal room environment or within the pool during the final block of hidden platform training as a function of the trial segment (.05 represents $0-0.5, .10$ represent $.06-.10$, etc. 


\section{Direct}

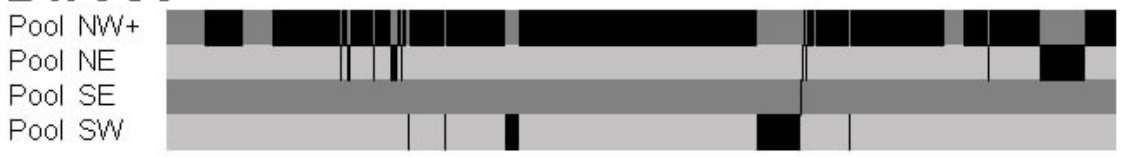

Distal NW+

ois

oceses

Distal SW

Figure 11.

\section{Non-direct}
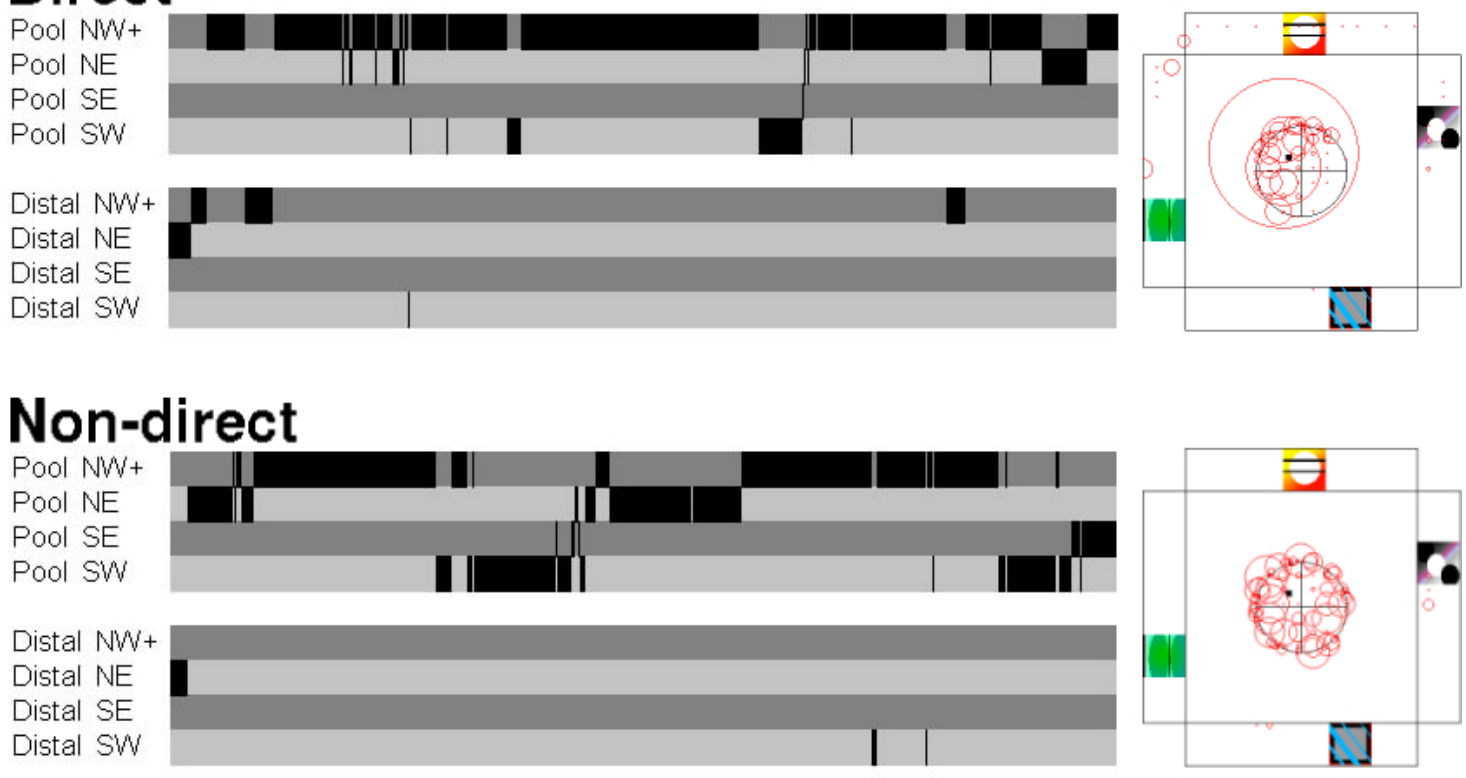

Figure 11: Representative ethogram (left) and dwell plot (right) during the no-platform probe trial for participants classified as direct or non-direct. The ethograms represent $60 \mathrm{sec}$ of navigation and follow the same conventions described in Fig. 9. The dwell plots were created by taking individual regions in the room on a grid comprised .25X.25 unit sections. The center of the red circles represents the location of the eye position in 3-d space and the diameter of the circle represents the total amount of time during the probe trial the eyes were positioned at that location. Because the duration of the trial was identical for both participants shown here, the relative diameters of the circles can be directly compared. The large circle shown for the direct participant is located in the target quadrant $(\mathrm{NW})$. 
Figure 12.
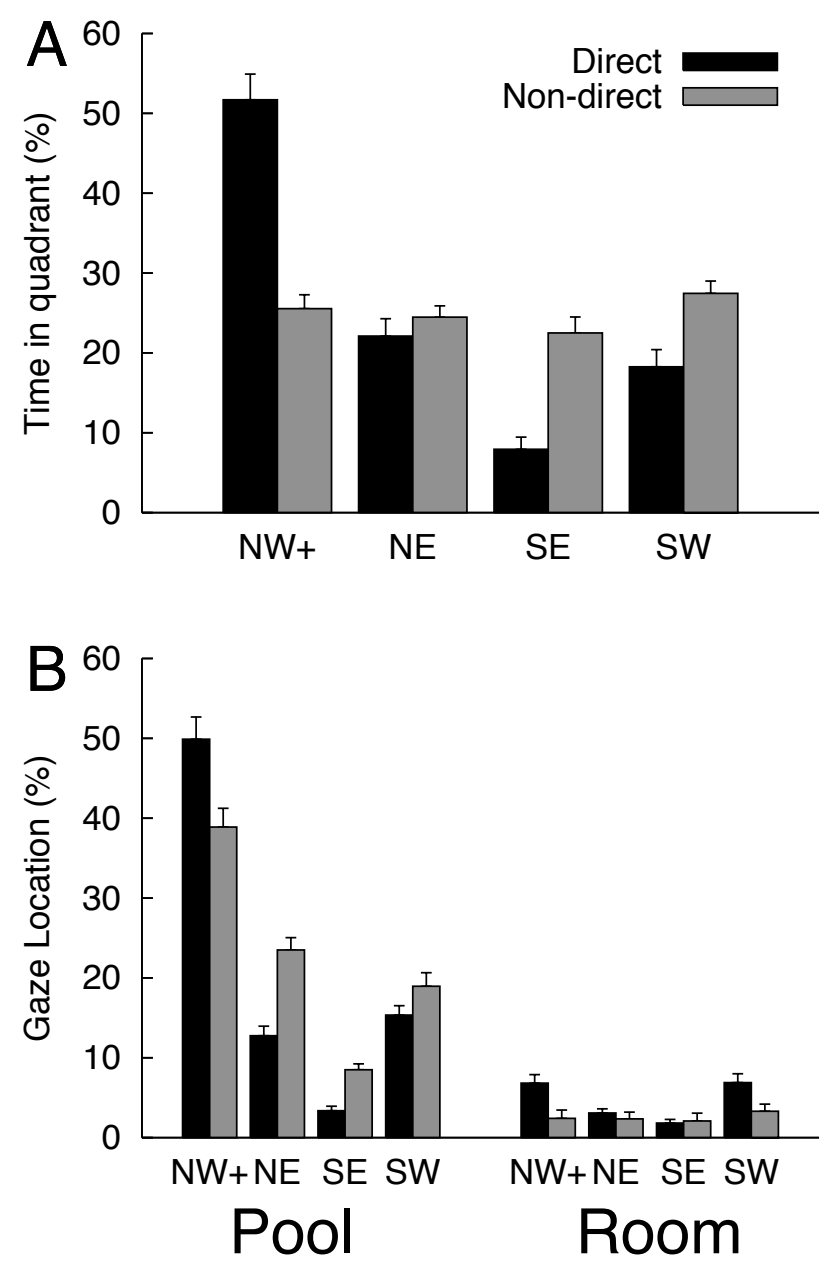

Figure 12: A) Mean percentage (+SEM) time that direct and non-direct participants spent in each quadrant of the pool during the no-platform probe trial. B) Mean percentage (+SEM) time that direct and non-direct participants spent looking in each of 8 regions of environment (4 quadrants in the pool and 4 quadrants in the distal room). The platform was located in the NW quadrant of the pool during training. 